The Astrophysical Journal, 689, 2008 DeCEMber 10, IN PRESS

Preprint typeset using $\mathrm{LT}_{\mathrm{E}} \mathrm{X}$ style emulateapj v. 03/07/07

\title{
TURBULENCE-DRIVEN POLAR WINDS FROM T TAURI STARS ENERGIZED BY MAGNETOSPHERIC ACCRETION
}

\author{
STEVEN R. CRANMER \\ Harvard-Smithsonian Center for Astrophysics, 60 Garden Street, Cambridge, MA 02138 \\ Submitted 2008 July 8; accepted 2008 August 15 \\ Draft version August 30, 2021
}

\begin{abstract}
Pre-main-sequence stars are observed to be surrounded by both accretion flows and some kind of wind or jet-like outflow. Recent work by Matt and Pudritz has suggested that if classical T Tauri stars exhibit stellar winds with mass loss rates about 0.1 times their accretion rates, the wind can carry away enough angular momentum to keep the stars from being spun up unrealistically by accretion. This paper presents a preliminary set of theoretical models of accretion-driven winds from the polar regions of $\mathrm{T}$ Tauri stars. These models are based on recently published self-consistent simulations of the Sun's coronal heating and wind acceleration. In addition to the convection-driven MHD turbulence (which dominates in the solar case), we add another source of wave energy at the photosphere that is driven by the impact of plasma in neighboring flux tubes undergoing magnetospheric accretion. This added energy, determined quantitatively from the far-field theory of MHD wave generation, is sufficient to produce $\mathrm{T}$ Tauri-like mass loss rates of at least 0.01 times the accretion rate. While still about an order of magnitude below the level required for efficient angular momentum removal, these are the first self-consistent models of $\mathrm{T}$ Tauri winds that agree reasonably well with a range of observational mass loss constraints. The youngest modeled stellar winds are supported by Alfvén wave pressure, they have low temperatures ("extended chromospheres"), and they are likely to be unstable to the formation of counterpropagating shocks and clumps far from the star.
\end{abstract}

Subject headings: accretion, accretion disks — stars: coronae — stars: mass loss — stars: pre-main sequence — stars: winds, outflows — turbulence

\section{INTRODUCTION}

Our current state of knowledge about how stars and planets are formed comes from an intertwined web of observations (spanning the electromagnetic spectrum) and theoretical work. The early stages of low-mass star formation comprise a wide array of inferred physical processes, including disk accretion, various kinds of outflow, and magnetohydrodynamic (MHD) activity on time scales ranging from hours to millennia (see, e.g., Lada 1985; Bertout 1989; Appenzeller \& Mundt 1989; Hartmann 2000; Königl \& Pudritz 2000; McKee \& Ostriker 2007; Shu et al. 2007). A key recurring issue is that there is a great deal of mutual interaction and feedback between the star and its circumstellar environment. This interaction can determine how rapidly the star rotates, how active the star appears from radio to X-ray wavelengths, and how much mass and energy the star releases into its interplanetary medium.

A key example of circumstellar feedback is the magnetospheric accretion paradigm for classical T Tauri stars (Lynden-Bell \& Pringle 1974; Uchida \& Shibata 1984; Camenzind 1990; Königl 1991). Because of strong ( 1 kG) stellar magnetic fields, the evolving equatorial accretion disk does not penetrate to the stellar surface, but instead is stopped by the stellar magnetosphere. Accretion is thought to proceed via ballistic infall along magnetic flux tubes threading the inner disk, leading to shocks and hot spots on the surface. The primordial accretion disk is dissipated gradually as the star enters the weak-lined T Tauri star phase, with a likely transition to a protoplanetary dust/debris disk.

Throughout these stages, solar-mass stars are inferred to exhibit some kind of wind or jet-like outflow. There are several possible explanations of how and where the outflows arise, including extended disk winds, $\mathrm{X}$-winds, impulsive (plasmoidlike) ejections, and "true" stellar winds (e.g., Paatz \& Camen- zind 1996; Calvet 1997; Königl \& Pudritz 2000; Dupree et al. 2005; Edwards et al. 2006; Ferreira et al. 2006; Gómez de Castro \& Verdugo 2007; Cai et al. 2008). Whatever their origin, the outflows produce observational diagnostics that indicate mass loss rates exceeding the Sun's present mass loss rate by factors of $10^{3}$ to $10^{6}$. It is of some interest to evaluate how much of the observed outflow can be explained solely with stellar winds, since these flows are locked to the star and thus are capable of removing angular momentum from the system. Recent work by Matt \& Pudritz $(2005,2007,2008)$ has suggested that if there is a stellar wind with a sustained mass loss rate about $10 \%$ of the accretion rate, the wind can carry away enough angular momentum to keep $\mathrm{T}$ Tauri stars from being spun up unrealistically by the accretion.

Despite many years of study, the dominant physical processes that accelerate winds from cool stars have not yet been identified conclusively. For many stars, the large-scale energetics of the system-i.e., the luminosity and the gravitational potential - seem to determine the overall magnitude of the mass loss (Reimers 1975; 1977; Schröder \& Cuntz 2005). Indeed, for the most luminous cool stars, radiation pressure seems to provide a direct causal link between the luminosity $L_{*}$ and the mass loss rate $\dot{M}_{\text {wind }}$ (e.g., Gail \& Sedlmayr 1987; Höfner 2005). However, for young solar-mass stars, other mediating processes (such as coronal heating, waves, or time-variable magnetic ejections) are more likely to connect the properties of the stellar interior to the surrounding outflowing wind. For example, magnetohydrodynamic (MHD) waves have been studied for several decades as a likely way for energy to be transferred from late-type stars to their winds (Hartmann \& MacGregor 1980; DeCampli 1981; Airapetian et al. 2000; Falceta-Gonçalves et al. 2006; Suzuki 2007).

In parallel with the above work in improving our understanding of stellar winds, there has been a great deal of 


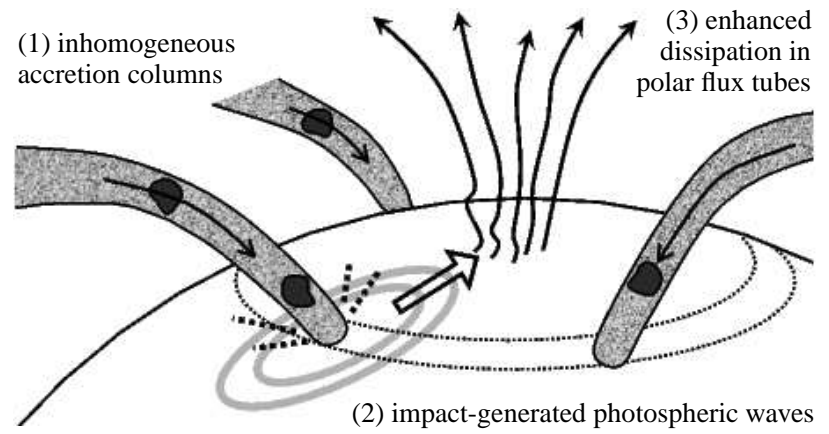

FIG. 1.- Summary sketch of the accretion and wind geometry discussed in this paper. (1) Magnetospheric accretion streams are assumed to impact the star at mid-latitudes. (2) Dense clumps in the accretion streams generate MHD waves that propagate horizontally over the stellar surface. (3) Polar magnetic field lines exhibit enhanced photospheric wave activity and thus experience stronger coronal heating and stellar wind acceleration.

progress toward identifying and characterizing the processes that produce the Sun's corona and wind. It seems increasingly clear that closed magnetic loops in the low solar corona are heated by small-scale, intermittent magnetic reconnection that is driven by the continual stressing of their footpoints by convective motions (e.g., Aschwanden 2006; Klimchuk 2006). The open field lines that connect the Sun to interplanetary space, though, appear to be energized by the dissipation of waves and turbulent motions that originate at the stellar surface (Tu \& Marsch 1995; Cranmer 2002; Suzuki 2006; Kohl et al. 2006). Parker's (1958) classic paradigm of solar wind acceleration via gas pressure in a hot $\left(T \sim 10^{6} \mathrm{~K}\right)$ corona still seems to be the dominant mechanism, though waves and turbulence have an impact as well. A recent self-consistent model of turbulence-driven coronal heating and solar wind acceleration has succeeded in reproducing a wide range of observations with no ad-hoc free parameters (Cranmer et al. 2007). This progress on the solar front is a fruitful jumpingoff point for a better understanding of the basic physics of winds and accretion in young stars.

The remainder of this paper is organized as follows. $\S 2$ presents an overview of the general scenario of accretiondriven MHD waves that is proposed here to be important for driving $\mathrm{T}$ Tauri mass loss. In $\S 3$ the detailed properties of an evolving solar-mass star are presented, including the fundamental stellar parameters, the accretion rate and disk geometry, and the properties of the clumped gas in the magnetospheric accretion streams. These clumped streams impact the stellar surface and create MHD waves that propagate horizontally to the launching points of stellar wind streams. $\S 4$ describes how self-consistent models of these wind regions are implemented, and $\S 5$ gives the results. Finally, $\S 6$ contains a summary of the major results of this paper and a discussion of the implications these results may have on our wider understanding of low-mass star formation.

\section{BASAL WAVE DRIVING FROM INHOMOGENEOUS ACCRETION}

Figure 1 illustrates the proposed connection between accretion and stellar wind driving that is explored below. The models make use of the idea that magnetospheric accretion streams are likely to be highly unstable and time-variable, and thus much of the material deposited onto the star is expected to be in the form of intermittent dense clumps. The impact of each clump is likely to generate MHD waves on the stellar surface (Scheurwater \& Kuijpers 1988) and these waves can propagate across the surface, spread out the kinetic energy of accretion, and inject some of it into the global magnetic field. ${ }^{1}$ The models simulate the time-steady properties of the polar magnetic flux tubes, wherein the source of wave/turbulence energy at the photosphere comes from two sources: (1) accretion-driven waves that have propagated over the surface from the "hot spots," and (2) the ever-present sub-photospheric convection, which shakes the magnetic field lines even without accretion, and is the dominant source of waves for the present-day Sun.

There is substantial observational evidence that the accretion streams of classical T Tauri stars are clumped and inhomogeneous (see, e.g., Gullbring et al. 1996; Safier 1998; Bouvier et al. 2003, 2004, 2007; Stempels 2003). Strong variations in the accretion signatures take place over time scales ranging between a few hours (i.e., the free-fall time from the inner edge of the disk to the star) and a few days (i.e., the stellar rotation period). Some of this variability may arise from instabilities in the accretion shock (Chevalier \& Imamura 1982; Mignone 2005), with the possibility of transitions between stable and unstable periods of accretion (e.g., Romanova et al. 2008). Observations of the bases of accretion streams indicate the presence of turbulence (Johns \& Basri 1995) as well as discrete events that could signal the presence of magnetic reconnection (Giardino et al. 2007). Alternately, some of the observed variability may come from larger-scale instabilities in the torqued magnetic field that is threaded by the disk (e.g., Long et al. 2007; Kulkarni \& Romanova 2008) and which could also drive periodic reconnection events (van Ballegooijen 1994). The latter variations would not necessarily be limited to the rotation time scale, since "instantaneous" topology changes can happen much more rapidly.

The mechanisms by which impulsive accretion events (i.e., impacts of dense clumps) can create MHD waves on the stellar surface are described in more detail in $\S 3.4$. This paper uses the analytic results of Scheurwater \& Kuijpers (1988) to set the energy flux in these waves, based on the available kinetic energy in the impacts. It is useful to point out, however, that there are relatively well-observed examples of impulsegenerated waves on the Sun that help to justify the overall plausibility of this scenario. These are the so-called "Moreton waves" and "EIT waves" that are driven by solar flares and coronal mass ejections (CMEs). Moreton waves are localized ripples seen in the $\mathrm{H} \alpha$ chromosphere expanding out from strong flares and erupting filaments at speeds of 500 to $2000 \mathrm{~km} \mathrm{~s}^{-1}$ (Moreton \& Ramsey 1960). These seem to be consistent with fast-mode MHD shocks associated with the flaring regions (e.g., Uchida 1968; Balasubramaniam et al. 2007). A separate phenomenon, discovered more recently in the extreme ultraviolet and called EIT waves (Thompson et al. 1998), is still not yet well understood. These waves propagate more slowly than Moreton waves (typically 100 to 400 $\mathrm{km} \mathrm{s}^{-1}$ ), but they are often seen to traverse the entire diameter of the Sun and remain coherent. Explanations include fast-mode MHD waves (Wang 2000), solitons (Wills-Davey et al. 2007), and sheared current layers containing enhanced Joule heating (Delannée et al. 2008). These serve as ample evidence that impulsive phenomena can generate MHD fluctuations that travel across a stellar surface.

Finally, it is important to clarify some of the limitations of the modeling approach used in this paper. The models include

\footnotetext{
${ }^{1}$ A somewhat similar wave-generation scenario was suggested by Vasconcelos et al. (2002) and Elfimov et al. (2004), but their model was applied only to the energization of the accretion streams themselves, not to the regions exterior to the streams.
} 
only a description of the magnetospheric accretion streams (with an assumed dipole geometry) and the open flux tubes at the north and south poles of the star. Thus, there is no attempt to model either disk winds or the closed-field parts of the stellar corona, despite the fact that these are likely to contribute to many key observational diagnostics of T Tauri circumstellar gas. In addition, the accretion streams themselves are included only for their net dynamical impact on the polar (non-accreting) regions, and thus there is no need to describe, e.g., the temperature or ionization state inside the streams. The wind acceleration in the polar flux tubes is treated with a one-fluid, time-steady approximation similar to that described by Cranmer et al. (2007) for the solar wind. To summarize, this paper is a "proof of concept" study to evaluate how much about T Tauri outflows can be explained with only polar stellar winds that are energized by accretion-driven waves.

\section{BASELINE SOLAR-MASS EVOLUTION MODEL}

\subsection{Stellar Parameters and Accretion Rate}

To begin exploring how accretion-driven waves affect the winds of young stars, a representative evolutionary sequence of fundamental parameters was constructed for a solar-type star. The adopted parameters are not intended to reproduce any specific observation in detail and should be considered illustrative. The STARS evolution code ${ }^{2}$ (Eggleton 1971, 1972, 1973; Eggleton et al. 1973; Han et al. 1994; Pols et al. 1995) was used to evolve a star having a constant mass $M_{*}=1 M_{\odot}$ from the Hayashi track to well past the current age of the Sun. Neither mass accretion nor mass loss were included in the evolutionary calculation, and a standard solar abundance mixture ( $X=0.70, Z=0.02)$ was assumed. All other adjustable parameters of the code were held fixed at their default values.

Figure 2 presents a summary of the modeled stellar parameters. A Hertzsprung-Russell (H-R) diagram is shown in Figure $2 a$, with the bolometric luminosity $L_{*}$ plotted as a function of the effective temperature $T_{\text {eff }}$ for both the evolutionary model (with a subset of stellar ages $t$ indicated by symbols) and an approximate main sequence band for luminosity class V stars (de Jager \& Nieuwenhuijzen 1987). The current age of the Sun is denoted by $t=4.6 \times 10^{9} \mathrm{yr}$, or $\log _{10} t=9.66$. Figure $2 b$ shows the age dependence of a selection of relevant length scales, including the stellar radius $R_{*}$ and the photospheric pressure scale height $H_{*}$. The latter quantity is defined assuming a pure hydrogen gas, as

$$
H_{*}=\frac{k_{\mathrm{B}} T_{\mathrm{eff}}}{m_{\mathrm{H}}} \frac{R_{*}^{2}}{G M_{*}}
$$

where $k_{\mathrm{B}}$ is Boltzmann's constant, $m_{\mathrm{H}}$ is the mass of a hydrogen atom, and $G$ is the Newtonian gravitation constant. Note that for most of the applications below, only the relative variation of $H_{*}$ with age is needed and not its absolute value. The scale height is assumed to be proportional to the horizontal granulation scale length (e.g., Robinson et al. 2004), which in turn governs the perpendicular correlation length of Alfvénic turbulence in the photosphere (see $\S 4$ ).

The other major parameter to be specified as a function of age is the mass accretion rate $\dot{M}_{\text {acc }}$. For classical T Tauri stars, it is generally accepted that the accretion rate decreases with increasing stellar age $t$, but there is a large spread in measured values that may be affected by observational uncertainties in

2 The December 2003 version was obtained from:
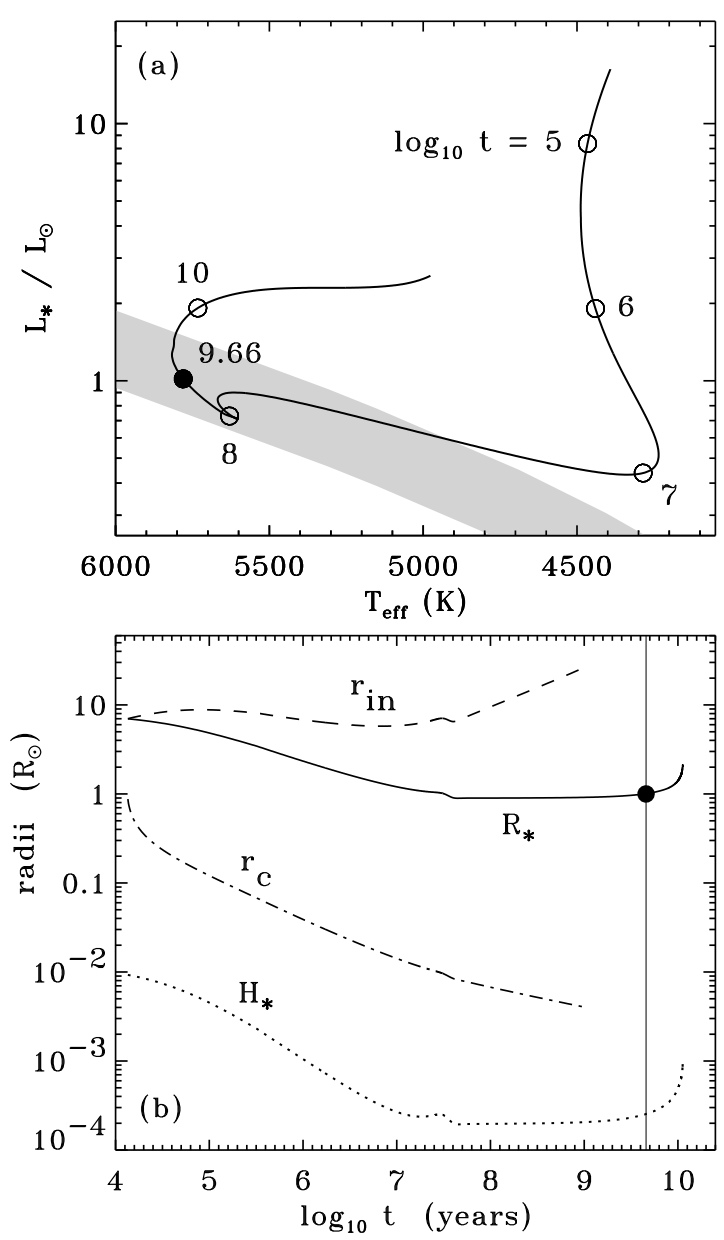

FIG. 2.- Basic properties of $1 M_{\odot}$ star. (a) H-R diagram showing the main sequence (gray band) and the modeled evolutionary track, with several representative ages (open circles) including the present-day Sun (filled circle). (b) Length scales, plotted in units of solar radii, as a function of age: stellar radius (solid line), inner edge of accretion disk (dashed line), accretion clump radius (dot-dashed line), and photospheric pressure scale height (dotted line).

both $\dot{M}_{\text {acc }}$ and $t$. In order to determine a representative age dependence for $\dot{M}_{\text {acc }}(t)$, we utilized tabulated accretion rates from Hartigan et al. (1995) and Hartmann et al. (1998), who interpreted optical/UV continuum excesses as an "accretion luminosity" that comes from the kinetic energy of accreted gas impacting the star. There is some overlap in the star lists from these two sources, and differences in the diagnostic techniques resulted in different values of both $\dot{M}_{\text {acc }}$ and $t$ for some stars common to both lists. Both values have been retained here, and thus Figure $3 a$ shows a total of 88 data points from both lists. ${ }^{3}$ The observational uncertainties are not shown; they may be as large as an order of magnitude in $\dot{M}_{\text {acc }}$ and a factor of $\sim 3$ in the age (see, e.g., Figure 3 of Muzerolle et al. 2000).

Highlighted in Figure $3 a$, as filled symbols, are a subset of stars that appear to be on the $1 M_{\odot}$ Hayashi track (i.e., they have $T_{\text {eff }}$ between about 4000 and $4500 \mathrm{~K}$ ). An initial attempt to fit these 35 stars to a power-law age dependence yielded

$$
\dot{M}_{\text {acc }} \approx 2.8 \times 10^{-8} M_{\odot} \mathrm{yr}^{-1}\left(\frac{t}{10^{6} \mathrm{yr}}\right)^{-1.22} .
$$

\footnotetext{
${ }^{3}$ This figure shows relatively nearby Galactic stars only. In the LMC, the mass accretion rates seem to be much higher at ages around $10 \mathrm{Myr}$ (e.g., Romaniello et al. 2004), which could indicate a substantial metallicity dependence for many properties of the $\mathrm{T}$ Tauri phase.
} 

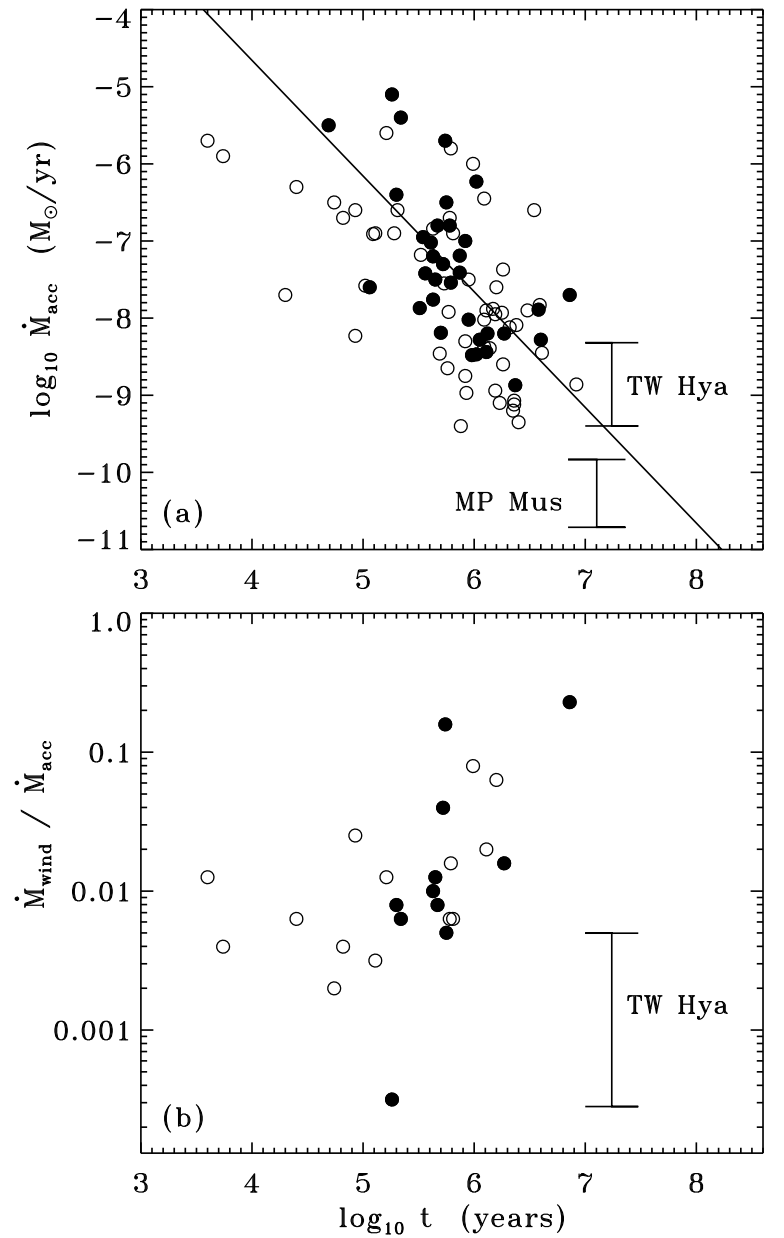

FIG. 3.- Mass accretion rates and mass loss rates for classical T Tauri stars. (a) Measured accretion rates from Hartigan et al. (1995) and Hartmann et al. (1998) (open circles) with the subset of roughly solar-mass stars highlighted (filled circles). The best-fit curve from eq. (3) is also shown (solid line) as well as two measured rates for the stars TW Hya and MP Mus (error bars); see text for details. (b) Ratio of mass loss rate to mass accretion rate for measured T Tauri stars, where the symbols have the same meanings as in panel (a).

However, the theoretical accretion disk models of Hartmann et al. (1998) found that the exponent $\eta$ in $\dot{M}_{\text {acc }} \propto t^{-\eta}$ is most likely to range between about 1.5 and 2.8 , with the lower end of that range being most likely. Thus, the fitted value of $\eta=$ 1.22 above was judged to be too low, and a more realistic fit was performed by fixing $\eta=1.5$ and finding

$$
\dot{M}_{\text {acc }} \approx 2.2 \times 10^{-8} M_{\odot} \mathrm{yr}^{-1}\left(\frac{t}{10^{6} \mathrm{yr}}\right)^{-1.5}
$$

which is used in the wind models below and is plotted in Figure $3 a$. The scatter around the mean fit curve has a roughly normal distribution (in the logarithm of $\dot{M}_{\text {acc }}$ ) with a standard deviation ranging between a factor of 6 (for the 35 solar-mass stars) to 8 (for all 88 data points) on either side of the representative trend given by equation (3). The sensitivity of the resulting accretion-driven wind to this scatter is explored further below in $\$ 5.4$.

Classical T Tauri magnetospheric accretion is believed to end at an age around 10 Myr (e.g., Strom et al. 1989; Bouvier et al. 1997). This is probably coincident with the time when the inner edge of the accretion disk grows to a larger extent than the Keplerian corotation radius, with "propellerlike" outflow replacing the accretion (Illarionov \& Sunyaev
1975; Ustyugova et al. 2006). Rotation is not included in the models presented here, so this criterion is not applied directly. However, for advanced ages (e.g., $t \gtrsim 100 \mathrm{Myr}$ ) equation (3) gives increasingly weak accretion rates that end up not having any significant effect on the stellar wind. Thus, even though the Figures below plot the various accretion-driven quantities up to an age of $1 \mathrm{Gyr}\left(\log _{10} t=9\right)$, the models below do not apply any abrupt cutoff to the accretion rate.

Figure $3 b$ plots mass loss rates $\dot{M}_{\text {wind }}$ for a subset of classical T Tauri stars that have measurements of blueshifted emission in forbidden lines such as [O I] $\lambda 6300$ (Hartigan et al. 1995). The mass loss rates are shown as an efficiency ratio $\dot{M}_{\text {wind }} / \dot{M}_{\text {acc }}$ which depends on the combined uncertainties of both the outflow and accretion properties. The largest ratio shown, at $\log _{10} t \approx 6.9$, is for the jet of HN Tau, and the original value from Hartigan et al. (1995) has been replaced with the slightly lower revised value from Hartigan et al. (2004). It is uncertain whether the measured outflows originate on the stellar surface or in the accretion disk (see, e.g., Paatz \& Camenzind 1996; Calvet 1997; Ferreira et al. 2006), but these rates can be used as upper limits for any stellar wind component.

Figure 3 also gives additional information about two of the oldest reported classical T Tauri stars: TW Hya and MP Mus. Note, however, that their extremely uncertain accretion rates were not included in the fitting for $\dot{M}_{\text {acc }}(t)$. The age of TW Hya is quite uncertain; it is often given as $\sim 10$ Myr (e.g., Muzerolle et al. 2000), but values as large as 30 Myr have been computed (Batalha et al. 2002) and the plotted error bar attempts to span this range. Similarly, the age of MP Mus seems to be between 7 and 23 Myr (e.g., Argiroffi et al. 2007). The plotted upper and lower limits on $\dot{M}_{\text {acc }}$ for TW Hya come from from Batalha et al. (2002) and Muzerolle et al. (2000), respectively, and the range of values for $\dot{M}_{\text {wind }}$ in Figure $3 b$ were taken from Dupree et al. (2005). The plotted ratio, however, divides the observational limits on $\dot{M}_{\text {wind }}$ by a mean value of $\dot{M}_{\text {acc }}=1.4 \times 10^{-9} M_{\odot} \mathrm{yr}^{-1}$, in order to avoid creating an unrealistically huge range. For MP Mus, the mean accretion rate of $5 \times 10^{-11} M_{\odot} \mathrm{yr}^{-1}$ was derived from X-ray measurements by Argiroffi et al. (2007). The plotted error bars for $\dot{M}_{\text {acc }}$ were estimated by using the $1 \sigma$ uncertainties on the X-ray emission measure and column density.

\subsection{Magnetospheric Accretion Streams}

The dynamical properties of accretion streams are modeled here using an axisymmetric dipole magnetic field, coupled with the assumption of ballistic infall (e.g., Calvet \& Hartmann 1992; Muzerolle et al. 2001). Although it is almost certain that the actual magnetic fields of T Tauri stars are not dipolar (Donati et al. 2007; Jardine et al. 2008), this assumption allows the properties of the accretion streams to be calculated simply and straightforwardly as a function of evolutionary age.

Stellar field lines that thread the accretion disk are bounded between inner and outer radii $r_{\text {in }}$ and $r_{\text {out }}$ as measured in the equatorial plane. We assume the inner radius - also called the "truncation radius"-is described by Königl's (1991) application of neutron-star accretion theory (see also Davidson \& Ostriker 1973; Elsner \& Lamb 1977; Ghosh \& Lamb 1979a,b). This expression for $r_{\text {in }}$ is given by determining where the magnetic pressure of the inner dipole region balances the gas pressure in the outer accretion region. Assuming free-fall in the 
accretion stream,

$$
r_{\mathrm{in}}=\beta_{\mathrm{GL}}\left(\frac{B_{*}^{4} R_{*}^{12}}{2 G M_{*} \dot{M}_{\mathrm{acc}}^{2}}\right)^{1 / 7}
$$

where the scaling constant $\beta_{\mathrm{GL}}$ describes the departure from ideal magnetostatic balance; i.e., $\beta_{\mathrm{GL}}=1$ gives the standard "Alfvén radius" at which the pressures balance. Following Königl (1991), the value $\beta_{\mathrm{GL}}=0.5$ is used here. The outer disk radius $r_{\text {out }}$ may be as large as the Keplerian corotation radius, but it may not extend that far in reality (e.g., Hartmann et al. 1994; Bessolaz et al. 2008). For simplicity, the outer disk radius is assumed to scale with the inner disk radius as

$$
r_{\text {out }}=r_{\text {in }}(1+\epsilon)
$$

where a constant value of $\epsilon=0.1$ was adopted. This value falls within the range of empirically constrained outer/inner disk ratios used by Muzerolle et al. (2001) to model $\mathrm{H} \alpha$ profiles; they used effective values of $\epsilon$ between 0.034 and 0.36 . The intermediate value of 0.1 produces reasonable magnitudes for the filling factors of accretion stream footpoints on the stellar surface (see below).

Note that equation (4) above requires the specification of the surface magnetic field strength $B_{*}$. In the models of the open flux tubes (in the polar regions) used below, a solartype magnetic field is adopted. The photospheric value of $B_{*} \approx 1500 \mathrm{G}$ is held fixed for the footpoints of the stellar wind flux tubes (see Cranmer \& van Ballegooijen 2005). For the mid-latitude field at the footpoints of the accretion streams, though, a slightly weaker field of $1000 \mathrm{G}$ is used.

Figure $2 b$ shows the resulting age dependence for $r_{\text {in }}$. For the youngest modeled "protostars" $\left(t \lesssim 10^{4} \mathrm{yr}\right)$, the accretion rate is so large that $r_{\text {in }}$ would be smaller than the stellar radius itself. In that case, the accretion disk would penetrate down to the star and there would be no magnetospheric infall. Thus, the youngest age considered for the remainder of this paper is $t=13.5$ kyr (i.e., $\log _{10} t=4.13$ ), for which $r_{\text {in }}=R_{*}$. Over most of the classical T Tauri age range (0.1-10 Myr), $r_{\text {in }}$ decreases slightly in absolute extent, but the ratio $r_{\text {in }} / R_{*}$ increases and is approximately proportional to $t^{0.2}$.

For any radius $r>R_{*}$ in the equatorial disk, an aligned dipole field line impacts the stellar surface at colatitude $\theta$, where $R_{*}=r \sin ^{2} \theta$. This allows the colatitudes $\theta_{\text {in }}$ and $\theta_{\text {out }}$ to be computed from $r_{\text {in }}$ and $r_{\text {out }}$, and these can be used to compute the fraction of stellar surface area $\delta$ that is filled by accretion streams, with

$$
\delta=\cos \theta_{\text {out }}-\cos \theta_{\text {in }}
$$

(see also Lamzin 1999). Note that both the northern and southern polar "rings" are taken into account in the above expression. The filling factor $\delta$ depends sensitively on the geometry of the accretion volume; i.e., for a dipole field, it depends only on the outer/inner radius parameter $\epsilon$ and the ratio $r_{\text {in }} / R_{*}$. Similarly, the fractional area subtended by both polar caps, to the north and south of the accretion rings, is given by

$$
\delta_{\text {pol }}=1-\cos \theta_{\text {out }} \text {. }
$$

Dupree et al. (2005) called this quantity $\phi$ and estimated it to be $\sim 0.3$ for TW Hya.

Accreting gas is assumed to be accelerated from rest at the inner edge of the disk, and thus is flowing at roughly the ballistic free-fall speed at the stellar surface,

$$
v_{\mathrm{ff}}=\left[\frac{2 G M_{*}}{R_{*}}\left(1-\frac{R_{*}}{r_{\mathrm{in}}}\right)\right]^{1 / 2} .
$$

This slightly underestimates the mean velocity, since in reality the streams come from radii between $r_{\text {in }}$ and $r_{\text {out }}$. Using this expression allows the ram pressure at the stellar surface to be computed, with

$$
P_{\mathrm{ram}}=\frac{\rho v_{\mathrm{ff}}^{2}}{2}=\frac{v_{\mathrm{ff}} \dot{M}_{\mathrm{acc}}}{8 \pi \delta R_{*}^{2}}
$$

(e.g., Hartmann et al. 1997; Calvet \& Gullbring 1998). The accretion is assumed to be stopped at the point where $P_{\text {ram }}$ is balanced by the stellar atmosphere's gas pressure. A representative $\mathrm{T}$ Tauri model atmosphere (with gray opacity and local thermodynamic equilibrium) was used to determine the height dependence of density and gas pressure. The atmospheric density $\rho_{\mathrm{sh}}$ was thus defined as that of the highest point in the atmosphere that remains undisturbed by either the shock or its (denser) post-shock cooling zone. The age dependence of $\rho_{\text {sh }}$ was computed from the condition of ram pressure balance. Examining these resulting values, though, yielded a useful approximation for this quantity. For most of the evolutionary ages considered for the solar-mass $\mathrm{T}$ Tauri star, the accretion is stopped a few scale heights above the photosphere, at which the temperature has reached its minimum radiative equilibrium value of $\sim 0.8 T_{\text {eff. }}$ This value can be used in the definition of gas pressure to obtain a satisfactory estimate for $\rho_{\mathrm{sh}}$; i.e., one can solve for

$$
\rho_{\mathrm{sh}} \approx \frac{5 m_{\mathrm{H}} P_{\mathrm{ram}}}{4 k_{\mathrm{B}} T_{\mathrm{eff}}}
$$

using equation (9) above and obtain a value within about $10 \%$ to $20 \%$ of the value interpolated from the full stellar atmosphere model. All of these values are only order-of-magnitude estimates, of course, since neither the post-shock heating nor the thermal equilibrium inside the accretion columns has been taken into account consistently.

In addition to the density at the shock, the models below also require specifying the density at the visible stellar surface. A representative photospheric mass density $\rho_{*}$ was computed from the criterion that the Rosseland mean optical depth should have a value of approximately one:

$$
\tau_{\mathrm{R}} \approx \kappa_{\mathrm{R}} \rho_{*} H_{*}=1
$$

where $H_{*}$ is the photospheric scale height as defined above and $\kappa_{\mathrm{R}}$ is the Rosseland mean opacity (in $\mathrm{cm}^{2} \mathrm{~g}^{-1}$ ) interpolated as a function of temperature and pressure from the table of Kurucz (1992). The resulting ratio $\rho_{\mathrm{sh}} / \rho_{*}$ varies strongly over the T Tauri evolution, from a value of order unity (for the youngest stars with the strongest accretion) down to $10^{-3}$ at the end of the accretion phase.

Figure 4 shows several of the accretion-stream quantities defined above. Figure $4 a$ shows the how the fractional area of both the accretion streams $(\delta)$ and the polar caps $\left(\delta_{\text {pol }}\right)$ decrease similarly with increasing age. Calvet \& Gullbring (1998) gave observationally determined values of $\delta$ for a number of T Tauri stars, but there was no overall trend with age; $\delta$ was distributed (apparently) randomly between values of about 0.001 and 0.1 for ages between $\log _{10} t=4$ and 7 . Dupree et al. (2005) estimated $\delta_{\text {pol }}$ to be about 0.3 , which agrees with the youngest $\mathrm{T}$ Tauri stars modeled here. Figure $4 b$ plots the age dependence of both the photospheric density $\rho_{*}$ and the density at the accretion shock $\rho_{\text {sh }}$ (computed both numerically and using eq. [10]).

\subsection{Properties of Inhomogeneous Accretion ("Clumps")}



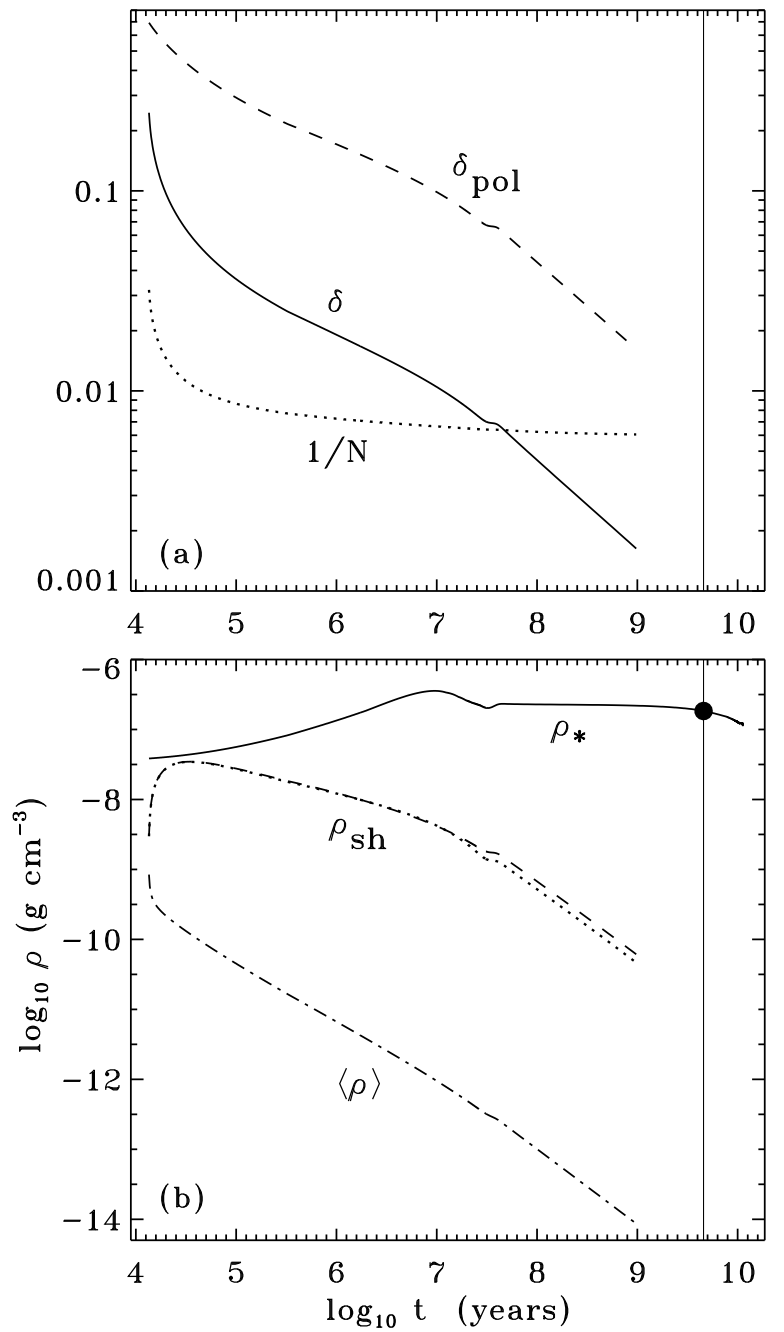

FIG. 4.- Accretion stream properties as a function of age. (a) Filling factor of accretion columns that thread the disk (solid line), filling factor of polar caps containing open field lines (dashed line), and the reciprocal of the number of inhomogeneous accretion tubes in one hemisphere (dotted line). (b) Mass densities at the stellar photosphere (solid line) and at the accretion shock (exact: dashed line, approximation from eq. [10]: dotted line), and mean pre-shock density in the accretion stream (dot-dashed line).

Observational evidence for intermittency and time variability in the accretion streams was discussed in $\S 2$. Here, the flow along magnetospheric accretion columns is modeled as an idealized "string of pearls," where infalling dense clumps (i.e., blobs or clouds) are surrounded by ambient gas of much lower density. The clumps are assumed to be roughly spherical in shape with roughly the same extent in latitude as the magnetic flux tubes connecting the star and accretion disk. Thus, once they reach the stellar surface, the clumps have a radius given by

$$
r_{c}=\frac{R_{*}\left(\theta_{\mathrm{in}}-\theta_{\mathrm{out}}\right)}{2}
$$

where the angles $\theta_{\text {in }}$ and $\theta_{\text {out }}$ must be expressed in radians. Figure $2 b$ shows how $r_{c}$ varies with age.

The dense clumps are assumed to impact the star with a velocity $v_{c}$ equivalent to the free-fall speed $v_{\text {ff }}$ defined in equation (8). Scheurwater \& Kuijpers (1988) defined a fiducial shock-crossing time for clumps impacting a stellar surface, with $t_{c}=1.5 r_{c} / v_{c}$. This time scale is of the same order of magnitude as the time it would take the blob to pass through the stellar surface if it was not stopped. It is useful to assume that the clumps are spread out along the flux tube with a constant periodicity; i.e., that along a given flux tube, clumps impact the star at a time interval $\Delta t=\zeta t_{c}$, where the dimensionless intermittency factor $\zeta$ must be larger than 1 .

When considering the mass accreted by infalling clumps, we follow Scheurwater \& Kuijpers (1988) and define the density interior to each clump as $\rho_{c}$ and the (lower) ambient interclump density as $\rho_{0}$. For the purpose of mass flux conservation along the magnetic flux tubes, these densities are defined at the stellar surface. Despite the fact that the ratio $\rho_{c} / \rho_{0}$ appears in several resulting quantities below, it always is canceled out by other quantities that depend separately on $\rho_{c}$ and $\rho_{0}$ such that the numerical value of their ratio never needs to be specified directly. A more relevant quantity for accretion is the mean density $\langle\rho\rangle$ in the flux tube, which is by definition larger than $\rho_{0}$ and smaller than $\rho_{c}$. The clump "overdensity ratio" $\left(\rho_{c} /\langle\rho\rangle\right)$ can be computed by comparing the volume subtended by one clump with the volume traversed by a clump over time $\Delta t$. In other words, if one assumes that $\rho_{c} \gg \rho_{0}$, one can find the mean density $\langle\rho\rangle$ by spreading out the gas in each clump to fill its own portion of the flux tube. Thus,

$$
\frac{\rho_{c}}{\langle\rho\rangle}=\frac{v_{c} \Delta t \pi r_{c}^{2}}{4 \pi r_{c}^{3} / 3}=\frac{9 \zeta}{8} \text {. }
$$

The overdensity ratio (or, equivalently, $\zeta$ ) is a free parameter of this system, and a value of $\rho_{c} /\langle\rho\rangle=3$ was chosen more or less arbitrarily. This sets $\zeta=24 / 9$. Below, the resulting Alfvén wave amplitude (caused by the periodic clump impacts) is found to depend on an overall factor of $\zeta^{1 / 2}$; i.e., it is relatively insensitive to the chosen value of the overdensity ratio.

In order to relate the inhomogeneous properties along a given flux tube to the total mass accretion rate $\dot{M}_{\text {acc }}$, the total number of flux tubes impacting the star must be calculated. The quantity $N$ is defined as the number of flux tubes in either the northern or southern hemisphere that contain accreting clumps. This definition is specific to the assumption of an aligned dipole magnetic field, and it is convenient because the summed effect of infalling clumps measured at the north [south] pole is assumed to depend only on the flux tubes in the northern [southern] hemisphere. The total number of flux tubes impacting the star is thus assumed to be $2 N$. One can compute $N$ by comparing two different ways of expressing the mass accretion rate. First, we know that in a time-averaged sense, mass is accreted with a local flux $\langle\rho\rangle v_{c}$ over a subset of the stellar surface $\delta$ given by equation (6). Thus,

$$
\dot{M}_{\mathrm{acc}}=4 \pi \delta R_{*}^{2}\langle\rho\rangle \nu_{c} .
$$

Alternately, the mass in the $2 N$ flux tubes can be summed up by knowing that each clump deposits a mass $m_{c}=4 \pi r_{c}^{3} \rho_{c} / 3$, with

$$
\dot{M}_{\mathrm{acc}}=\frac{2 N m_{c}}{\Delta t}=\frac{16 \pi N}{9 \zeta} \rho_{c} v_{c} r_{c}^{2} .
$$

Equations (14) and (15) must give the same total accretion rate, so equating them gives a useful expression for

$$
N=2 \delta\left(\frac{R_{*}}{r_{c}}\right)^{2}
$$

where equation (13) was also used. This quantity is used below to compute the total effect of waves at the poles from the individual impact events. 
Figure $4 a$ shows $N^{-1}$ (rather than $N$ itself, to keep it on the same scale as the other plotted quantities), and it is interesting that $N$ remains reasonably constant around $100-150$ over most of the classical T Tauri phase of evolution. This mean value (for any instant of time) is large, but it is not so large that any fluctuations around the mean would be unresolvable due to averaging over the star. If the distribution of flux tubes is assumed to follow some kind of Poisson-like statistics, a standard deviation of order $N^{1 / 2}$ would be expected. In other words, for $N \approx 100$ there may always be something like a $10 \%$ level of fluctuations in the magnetospheric accretion rate.

The equations above also allow $\langle\rho\rangle$ and $\rho_{c}$ to be computed from the known accretion rate $\dot{M}_{\text {acc }}$. Figure $4 b$ shows $\langle\rho\rangle$ to typically be several orders of magnitude smaller than both $\rho_{\text {sh }}$ and $\rho_{*}$. This large difference arises because the ram pressure inside the accretion stream is much larger than the gas pressure in the stream. The ratio $\langle\rho\rangle / \rho_{\mathrm{sh}}$ at the stellar surface is given very roughly by $\left(c_{s} / v_{c}\right)^{2}$, where $c_{s}$ is the sound speed corresponding to $T_{\text {eff }}$, and $c_{s} \ll v_{c}$. For the youngest $\mathrm{T}$ Tauri stars modeled, however, $c_{s} \approx v_{c}$ and thus $\langle\rho\rangle \approx \rho_{\mathrm{sh}}$.

\subsection{Properties of Impact-Generated Waves}

This section utilizes the results of Scheurwater \& Kuijpers (1988), who computed the flux of magnetohydrodynamic (MHD) waves that arise from the impact of a dense clump onto a stellar surface. Scheurwater \& Kuijpers (1988) derived the total energy released in both Alfvén and fast-mode MHD waves from such an impact in the "far-field" limit (i.e., at horizontal distances large compared to the clump size $r_{c}$ ). Slow-mode MHD waves were not considered because of the use of the cold-plasma approximation. The models below utilize only their Alfvén wave result, since the efficiency of fastmode wave generation was found to be significantly lower than for Alfvén waves. Also, due to their compressibility, the magnetosonic (fast and slow mode) MHD waves are expected to dissipate more rapidly than Alfvén waves in stellar atmospheres (e.g., Kuperus et al. 1981; Narain \& Ulmschneider 1990, 1996; Whang 1997). Thus, even if fast-mode and Alfvén waves were generated in equal amounts at the impact site, the fast-mode waves may not survive their journey to the polar wind-generation regions of the star without being strongly damped.

For simplicity, we assume that waves propagate away from the impact site with an overall energy given by the Scheurwater \& Kuijpers (1988) Alfvén wave result, and that the waves undergo negligible dissipation. The wave energy released in one impact event is given by

$$
E_{\mathrm{A}}=0.06615 \frac{\rho_{c}}{\rho_{0}}\left(\frac{v_{c}}{V_{\mathrm{A}}}\right)^{3} m_{c} v_{c}^{2}
$$

where the numerical factor in front was given approximately as 0.07 in equation (57) of Scheurwater \& Kuijpers (1988), but has been calculated more precisely from their Besselfunction integral. In this context, the Alfvén speed $V_{\mathrm{A}}$ is defined as that of the ambient medium, with

$$
V_{\mathrm{A}}=\frac{B_{0}}{\sqrt{4 \pi \rho_{0}}}
$$

and $B_{0} \approx 1000 \mathrm{G}$ is the ambient magnetic field strength at the stellar surface, where the accretion streams connect with the star. Scheurwater \& Kuijpers (1988) assumed that $v_{c}<V_{\mathrm{A}}$ (i.e., that the impacting clumps do not strongly distort the background magnetic field). This tends to lead to a very low efficiency of wave generation, such that equation (17) may be considered a "conservative" lower limit to the available energy of fluctuations. For a given accretion column, the wave power that is emitted continuously by a stream of clumps is given by $E_{\mathrm{A}} / \Delta t$.

In order to compute the wave energy density at other points on the stellar surface, we make the assumption that the waves propagate out horizontally from the impact point. It is important to note that ideal Alfvén waves do not propagate any energy perpendicularly to the background magnetic field. There are several reasons, however, why this does not disqualify the adopted treatment of wave energy propagation over the horizontal stellar surface. First, the true evolution of the impact pulse is probably nonlinear. Much like the solar Moreton and EIT waves discussed in $\S 2$, nonlinear effects such as mode coupling, shock steepening, and soliton-like coherence are likely to be acting to help convey the total "fluctuation yield" of the impact across the stellar surface. Second, for any real star, the background magnetic field is never completely radial, and it will always have some component horizontal to the surface (along which even linear Alfvén waves can propagate). Thus, the dominant end-result of multiple cloud impacts is assumed here to be some kind of transverse field-line perturbations that are treated for simplicity with the energetics of Alfvén waves.

Considering waves that spread out in circular ripples from the impact point, the goal is to compute the horizontal wave flux (power per unit area) at a distance $x$ away from the central point. For this purpose, the stellar surface can be treated as a flat plane. The wave power is assumed to be emitted into an expanding cylinder with an approximate height of $2 r_{c}$ (the clump diameter) and a horizontal radius $x$. The horizontal flux $F_{\mathrm{A}}$ of waves into the vertical wall of the cylinder is given by dividing the power by the area of the surrounding wall, with

$$
F_{\mathrm{A}}=\frac{E_{\mathrm{A}} / \Delta t}{4 \pi x r_{c}}=0.0147 \frac{r_{c} \rho_{c}^{2} v_{c}^{3}}{x \rho_{0} \zeta}\left(\frac{v_{c}}{V_{\mathrm{A}}}\right)^{3} .
$$

Note that the flux decreases linearly with increasing $x$, as is expected for a cylindrical geometry.

The total wave flux from the effect of multiple clump impacts is computed at the north or south pole of the star. The accretion stream impact points are assumed to be distributed circularly in a ring around the pole. Thus, using the geometric quantities derived earlier, this implies $x=R_{*}\left(\theta_{\text {in }}+\theta_{\text {out }}\right) / 2$. Also, because each accretion column is assumed to be at the same horizontal distance from the target point at the pole, the total wave flux is given straightforwardly by $N$ times the flux due to one stream of clumps. The total accretion-driven wave flux arriving at either the north or south pole is thus given by $F_{\mathrm{A} \text {,tot }}=N F_{\mathrm{A}}$. It is assumed that the waves do not damp appreciably between where they are generated (at the bases of the accretion streams) and their destination (at the pole).

The standard definition of the flux of Alfvén waves, in a medium where the bulk flow speed is negligible in comparison to the Alfvén speed, is

$$
F_{\mathrm{A}, \text { tot }}=\rho_{0} v_{\perp}^{2} V_{\mathrm{A}} .
$$

This can be compared with the total wave flux derived above to obtain the perpendicular Alfvén wave velocity amplitude $v_{\perp}$. In units of the clump infall velocity $v_{c}$, the wave amplitude is thus given by

$$
\frac{v_{\perp}}{v_{c}}=0.1715 \frac{\rho_{c}}{\rho_{0}}\left(\frac{N r_{c}}{\zeta x}\right)^{1 / 2}\left(\frac{v_{c}}{V_{\mathrm{A}}}\right)^{2} .
$$


Note that there is no actual dependence on $\rho_{0}$, since the explicit factor of $\rho_{0}$ in the denominator is canceled by the density dependence of $V_{\mathrm{A}}^{-2}$.

The wave amplitude derived in equation (21) is the value at the shock impact height, which formally can be above or below the photosphere. The stellar wind models below, though, require the Alfvén wave amplitude to be specified exactly at the photosphere. If we assume that the wave energy density $U_{\mathrm{A}}=\rho v_{\perp}^{2}$ is conserved between the shock height and the photosphere, then the densities at those heights can be used to scale one to the other, with

$$
v_{\perp *}=v_{\perp}\left(\frac{\rho_{\mathrm{sh}}}{\rho_{*}}\right)^{1 / 2}
$$

where $v_{\perp *}$ is the photospheric wave amplitude, and the densities $\rho_{*}$ and $\rho_{\text {sh }}$ were defined above in $\S 3.2$.

Although the overall energy budget of accretion-driven waves is treated under the assumption that the waves are Alfvénic, it is likely that the strongly nonlinear stream impacts also give rise to compressible waves of some kind. As mentioned above, the analysis of Scheurwater \& Kuijpers (1988) did not take account of slow-mode MHD waves that, for parallel propagation and a strong background field, are identical to hydrodynamic acoustic waves. There is evidence, however, that another highly nonlinear MHD phenomenon-turbulent subsurface convection-gives rise to both longitudinal (compressible) and transverse (incompressible) MHD waves with roughly comparable energy densities (e.g., Musielak \& Ulmschneider 2002). Thus, the models below are given a photospheric source of accretion-driven acoustic waves that are in energy equipartition with the accretion-driven Alfvén waves; i.e., $U_{s}=U_{\mathrm{A}}$. The upward flux of acoustic waves is thus given by $F_{s}=c_{s} U_{s}$, where $c_{s}$ is the sound speed appropriate for $T_{\text {eff }}$.

For both the acoustic and Alfvén waves at the photosphere, the accretion-driven component is added to the intrinsic (convection-driven) component. A key assumption of this paper is that the convection-driven component is held fixed, as a function of age, at the values used by Cranmer et al. (2007) for the present-day Sun. This results in minimum values for the Alfvén wave amplitude $\left(0.255 \mathrm{~km} \mathrm{~s}^{-1}\right)$ and the acoustic wave flux $\left(10^{8} \mathrm{erg} \mathrm{cm}^{-2} \mathrm{~s}^{-1}\right)$ below which the models never go. There are hints that rapidly rotating young stars may undergo more intense subsurface convection than the evolved slowly rotating Sun (Käpylä et al. 2007; Brown et al. 2007; Ballot et al. 2007), but the implications of these additional variations with age are left for future work (see also $\S 6$ ).

Figure 5 displays various velocity quantities used in the accretion-driven wave scenario. The clump free-fall speed $v_{c}=v_{\mathrm{ff}}$ always remains smaller than the ambient Alfvén speed $V_{\mathrm{A}}$, which was an assumption that Scheurwater \& Kuijpers (1988) had to make in order for the background magnetic field to remain relatively undisturbed by the clumps. The accretion-driven Alfvén wave amplitude at the shock is larger than that measured at the photosphere (see eq. [22]), and at ages later than about 60 Myr the accretion-driven waves at the photosphere grow weak enough to be overwhelmed by the convection-driven waves. For a limited range of younger ages $\left(2 \times 10^{4}<t<5 \times 10^{5} \mathrm{yr}\right)$ the Alfvén wave motions are supersonic in the photosphere, with a peak value of $v_{\perp *}=18 \mathrm{~km}$ $\mathrm{s}^{-1}$ at $t=5 \times 10^{4} \mathrm{yr}$. The sharp decrease in wave amplitude at the youngest ages is due to the inner edge of the accretion disk coming closer to the stellar surface. In that limit, the ballistic infall speed $v_{\mathrm{ff}}$ becomes small and the latitudinal distance $x$ traversed by the waves becomes large, thus leaving negligible

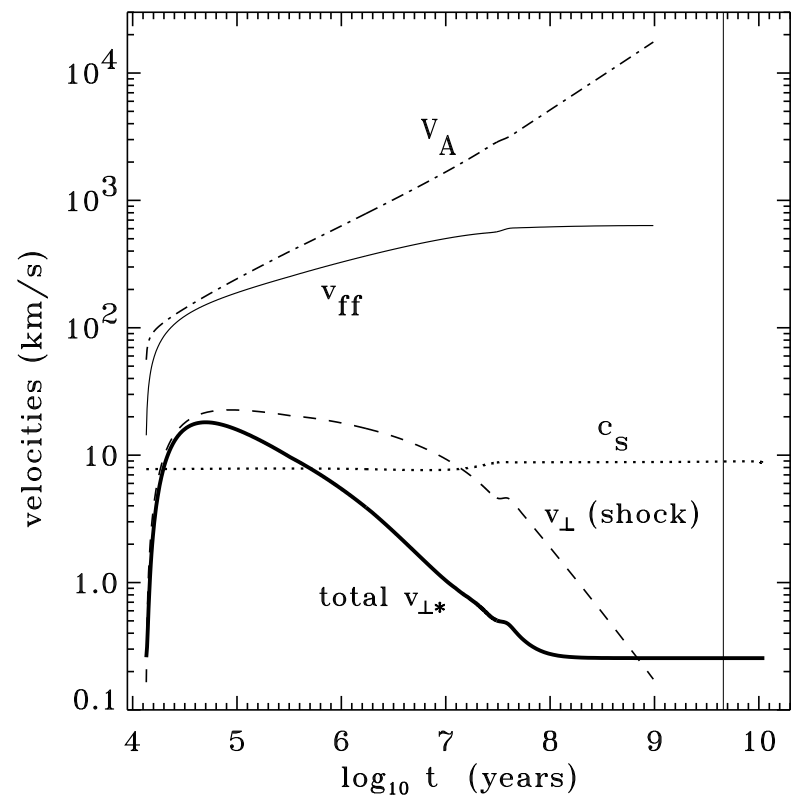

FIG. 5.- Velocities related to accretion streams as a function of age: freefall clump speed (thin solid line), ambient Alfvén speed (dot-dashed line), photospheric sound speed (dotted line). Plotted Alfvén wave amplitudes are those due to accretion-streams and measured at the shock (dashed line) and those due to both accretion and convection, measured at the photosphere (thick solid line).

energy in the waves once they reach the pole.

Finally, it is worthwhile to sum up how the above values depend on the relatively large number of assumptions made about the accretion streams. First, the use of an aligned dipole field for the accretion streams, the Königl (1991) expression for $r_{\text {in }}$, and the "string of pearls" model of clumps along the flux tubes are all somewhat simplistic and should be replaced by more realistic conditions in future work. Second, there are three primary parameters that had to be specified in order to determine numerical values for the accretion properties. These are: (1) the relative size of the outer disk radius with respect to the inner disk radius; i.e., $\epsilon=0.1$, (2) the clump overdensity ratio $\rho_{c} /\langle\rho\rangle=3$, and (3) the magnetic field strength at the base of the accretion streams $B_{0}=1000 \mathrm{G}$. Third, probably the most idealistic assumption in the modeled evolutionary sequence is the use of a single monotonic relation for $\dot{M}_{\text {acc }}$ versus $t$ (e.g., eq. [3]). $\S 6$ describes how these assumptions can be relaxed in subsequent modeling efforts.

\section{IMPLEMENTATION IN STELLAR WIND MODELS}

The steady-state outflow models presented here are numerical solutions to one-fluid conservation equations for mass, momentum, and energy along a polar flux tube. For the specific case of the solar wind, Cranmer et al. (2007) presented these equations and described their self-consistent numerical solution using a computer code called ZEPHYR. The T Tauri wind models are calculated with an updated version of ZEPHYR, with specific differences from the solar case described below.

\subsection{Conservation Equations and Input Physics}

The equation of mass conservation along a magnetic flux tube is

$$
\frac{1}{A} \frac{\partial}{\partial r}(\rho u A)=0
$$

where $u$ and $\rho$ are the wind speed and mass density specified as functions of radial distance $r$, and $A$ is the cross-sectional 
area of the polar flux tube. Magnetic flux conservation demands that the product $B_{0} A$ is constant along the flux tube, where $B_{0}(r)$ is the field strength that is specified explicitly.

The equation of momentum conservation is

$$
u \frac{\partial u}{\partial r}+\frac{1}{\rho} \frac{\partial P}{\partial r}=-\frac{G M_{*}}{r^{2}}+D
$$

where $P$ is the gas pressure and $D$ is the bulk acceleration on the plasma due to wave pressure; i.e., the nondissipative net ponderomotive force due to wave propagation through the inhomogeneous medium (Bretherton \& Garrett 1968; Belcher 1971; Jacques 1977). A complete expression for $D$ in the presence of damped acoustic and Alfvén waves was given by Cranmer et al. (2007). The simpler limit of wave pressure due to dissipationless Alfvén waves is discussed in more detail in $\S \S 5.2-5.3$.

For the pure hydrogen plasma assumed here, the gas pressure is given by $P=(1+x) \rho k_{\mathrm{B}} T / m_{\mathrm{H}}$, where $x$ is the hydrogen ionization fraction. Note that although the pressure is calculated for a hydrogen gas, the radiative cooling rate $Q_{\text {rad }}$ used in the energy equation is dominated by metals. ${ }^{4}$ Cranmer et al. (2007) tested the ZEPHYR code with two separate assumptions for the ionization balance. First, a self-consistent, but computationally intensive solution was used, which implemented a three-level hydrogen atom. In that model, the $n=1$ and $n=2$ levels were assumed to remain in relative local thermodynamic equilibrium (LTE) and the full rate equation between $n=2$ and the continuum was solved iteratively. Second, a simpler tabulated function of $x$ as a function of temperature $T$ was taken from a semi-empirical non-LTE model of the solar photosphere, chromosphere, and transition region (e.g., Avrett \& Loeser 2008). For both the solar and T Tauri star applications, the results for the two cases were extremely similar, and thus the simpler tabulated function was used in the models described below.

For solar-type winds, the key equation for both heating the corona and setting the mass loss rate is the conservation of internal energy,

$$
u \frac{\partial E}{\partial r}+\left(\frac{E+P}{A}\right) \frac{\partial}{\partial r}(u A)=Q_{\mathrm{rad}}+Q_{\mathrm{cond}}+Q_{\mathrm{A}}+Q_{\mathrm{S}}
$$

where $E$ is the internal energy density and the terms on the right-hand side are volumetric heating/cooling rates due to radiation, conduction, Alfvén wave damping, and acoustic (sound) wave damping. The terms on the left-hand side that depend on $u$ are responsible for enthalpy transport and adiabatic cooling. In a partially ionized plasma, the definition of $E$ is convention-dependent; we use the same one as Ulmschneider \& Muchmore (1986) and Mullan \& Cheng (1993), which is $E=(3 P / 2)+x \rho I_{\mathrm{H}} / m_{\mathrm{H}}$, where $I_{\mathrm{H}}=13.6 \mathrm{eV}$. The net heating/cooling from conduction $\left(Q_{\text {cond }}\right)$ is given by a gradual transition between classical Spitzer-Härm conductivity (when the electron collision rate is fast compared to the wind expansion rate) and Hollweg's $(1974,1976)$ prescription for free-streaming heat flux in the collisionless heliosphere (when electron collisions are negligible). All of these terms were described in more detail by Cranmer et al. (2007).

The volumetric radiative heating/cooling rate $Q_{\text {rad }}$ has been modified slightly from the earlier solar models. The solar rate is first computed as in Cranmer et al. (2007), but then it is

\footnotetext{
${ }^{4}$ Although this is formally inconsistent, the resulting properties of the plasma are not expected to be far from values computed with a more accurate equation of state.
}

multiplied by a correction factor similar to that suggested by Hartmann \& Macgregor (1980) for massive, optically thick chromospheres of late-type stars. The correction factor $f$ is assumed to be proportional to the escape probability $P_{\text {esc }}$ for photons in the core of the $\mathrm{Mg}$ II $\lambda 2803(h)$ and $\lambda 2796(k)$ lines. A simple expression that bridges the optically thin and thick limits, for a line with Voigt wings, is

$$
f=2 P_{\mathrm{esc}} \approx \frac{1}{1+\tau_{\mathrm{hk}}^{1 / 2}}
$$

(e.g., Mihalas 1978), which may err on the side of overestimating the escape probability and thus would give a conservative undercorrection to $Q_{\mathrm{rad}}$. Hartmann \& Macgregor (1980) assumed that the optical depth of low-ionization metals would scale as $g^{-1 / 2}$, where $g$ is the stellar surface gravity. Here, we compute the optical depth in the core of the $h$ and $k$ lines $\left(\tau_{\mathrm{hk}}\right)$ more exactly by integrating over the radial grid of density and temperature in the wind model,

$$
\tau_{\mathrm{hk}}=\int \chi_{\mathrm{hk}}\left(\frac{R_{*}}{r}\right)^{2} d r,
$$

where we include the spherical correction factor suggested by Lucy $(1971,1976)$ for extended atmospheres. The line-center extinction coefficient is given approximately by

$$
\chi_{\mathrm{hk}}=0.0153\left(\frac{n_{\mathrm{MgII}}}{10^{10} \mathrm{~cm}^{-3}}\right)\left(\frac{T}{10^{4} \mathrm{~K}}\right)^{-1 / 2} \mathrm{~cm}^{-1}
$$

and the number density $n_{\mathrm{Mg} \text { II }}$ of ground-state ions is given by the product of the hydrogen number density $\left(\rho / m_{\mathrm{H}}\right)$, the $\mathrm{Mg}$ abundance with respect to hydrogen $\left(3.4 \times 10^{-5}\right.$; Grevesse et al. 2007), and the ionization fraction of $\mathrm{Mg}^{+}$with respect to all $\mathrm{Mg}$ species. For temperatures in excess of $10^{4} \mathrm{~K}$, the latter is given by coronal ionization equilibrium (Mazzotta et al. 1998); for temperatures below $10^{4} \mathrm{~K}$, the coronal equilibrium curve is matched onto a relation determined from LTE Saha ionization balance.

Following Hartmann \& Macgregor (1980), the opacity correction factor $f$ is ramped up gradually as a function of temperature. When $T<7160 \mathrm{~K}, Q_{\text {rad }}$ is multiplied by the full factor $f$. For $7160<T<8440 \mathrm{~K}$, the multiplier is varied by taking $f^{(8440-T) / 1280}$, which ramps down to unity by the time the temperature rises to $8440 \mathrm{~K}$. For temperatures in excess of $8440 \mathrm{~K}$, the correction factor is not used.

The evolution and damping of Alfvén and acoustic waveswhich affects both $D$ in the momentum equation and $Q_{\mathrm{A}}$ and $Q_{\mathrm{S}}$ in the energy equation-is described in much more detail by Cranmer et al. (2007). Given a specified frequency spectrum of acoustic and Alfvén wave power in the photosphere, equations of wave action conservation are solved to determine the radial evolution of the wave amplitudes. Acoustic waves are damped by both heat conduction and entropy gain at shock discontinuities. Alfvén waves are damped by MHD turbulence, for which we only specify the net transport of energy from large to small eddies, assuming the cascade must terminate in an irreversible conversion of wave energy to heat.

Coupled with the wave action equations are also non-WKB transport equations to determine the degree of linear reflection of the Alfvén waves (e.g., Heinemann \& Olbert 1980). This is required because the turbulent dissipation rate depends on differences in energy density between upward and downward traveling waves (see also Matthaeus et al. 1999; Dmitruk et al. 2001, 2002; Cranmer \& van Ballegooijen 2005). The resulting values of the Elsasser amplitudes $Z_{ \pm}$, which denote the 
energy contained in upward $\left(Z_{-}\right)$and downward $\left(Z_{+}\right)$propagating waves, were then used to constrain the energy flux in the cascade. The Alfvén wave heating rate $\left(\mathrm{erg} \mathrm{s}^{-1} \mathrm{~cm}^{-3}\right)$ is given by a phenomenological relation that has evolved from analytic studies and numerical simulations; i.e.,

$$
Q_{\mathrm{A}}=\rho\left(\frac{1}{1+t_{\text {eddy }} / t_{\text {ref }}}\right) \frac{Z_{-}^{2} Z_{+}+Z_{+}^{2} Z_{-}}{4 \ell_{\perp}}
$$

(see also Hossain et al. 1995; Zhou \& Matthaeus 1990; Oughton et al. 2006). The transverse length scale $\ell_{\perp}$ is an effective perpendicular correlation length of the turbulence, and Cranmer et al. (2007) used a standard assumption that $\ell_{\perp}$ scales with the cross-sectional width of the flux tube (Hollweg 1986). The term in parentheses in equation (29) is an efficiency factor that accounts for situations in which the turbulent cascade does not have time to develop before the waves or the wind carry away the energy (Dmitruk \& Matthaeus 2003). The cascade is quenched when the nonlinear eddy time scale $t_{\text {eddy }}$ becomes much longer than the macroscopic wave reflection time scale $t_{\text {ref }}$.

\subsection{Model Inputs and Numerical Procedures}

The ZEPHYR code was designed to utilize as few free parameters as possible. For example, the coronal heating rate and the spatial length scales of wave dissipation are computed internally from straightforward physical principles and are not input as adjustable parameters. However, there are quantities that do need to be specified prior to solving the equations given in $\S 4.1$.

An important input parameter to ZEPHYR is the radial dependence of the background magnetic field $B_{0}(r)$. Although many stellar field-strength measurements have been made, relatively little is known about how rapidly $B_{0}$ decreases with increasing height above the photosphere, or how fragmented the flux tubes become on the granulation scale. Some information about this kind of structure is contained in "filling factors" that can be determined observationally (e.g., Saar 2001). However, these measurements may be biased toward the bright closed-field active regions and not the footpoints of stellar wind streams. Thus, as in other areas of this study, the present-day solar case was adopted as a baseline on which to vary the evolving properties of the $1 M_{\odot}$ model star.

The multi-scale polar coronal hole field of Cranmer \& van Ballegooijen (2005) was used as the starting point for the younger T Tauri models. The lower regions of that model were derived for a magnetostatic balance between the strongfield (low density) solar wind flux tube and the weak-field (high density) surrounding atmosphere. The radial dependence of magnetic pressure $B_{0}^{2} / 8 \pi$ thus scales with the gas pressure $P$. In the T Tauri models, then, the lower part of the magnetic field was stretched in radius in proportion with the pressure scale height $H_{*}$. Figure 6 shows $B_{0}(r)$ for several ages, along with an idealized dipole that does not take account of the lateral expansion of the flux tube close to the surface.

In addition to the global magnetic field strength, the ZEPHYR models also require three key wave-driving parameters to be specified at the lower boundary:

1. The photospheric acoustic flux $F_{s}$ mainly affects the heating at chromospheric temperatures $\left(T \sim 10^{4} \mathrm{~K}\right)$. The solar value of $10^{8} \mathrm{erg} \mathrm{cm}^{-2} \mathrm{~s}^{-1}$ was summed with the accretion-driven acoustic flux as discussed in $\S 3.4$. The power spectrum of acoustic waves was adopted from the solar spectrum given in Figure 3 of Cranmer

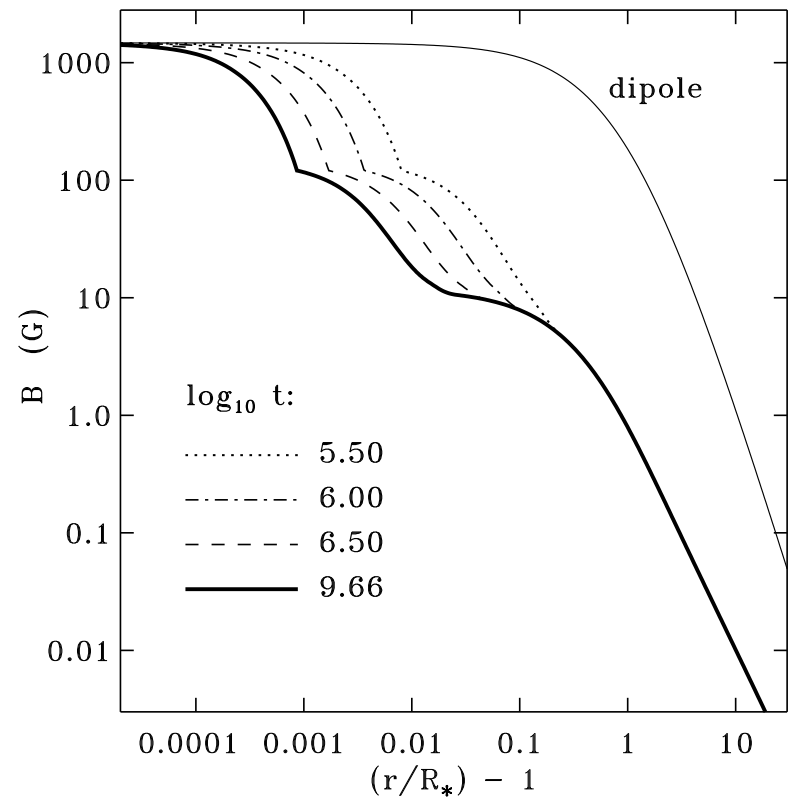

FIG. 6.- Background magnetic field $B_{0}$ over the stellar poles as a function of the height above the photosphere (measured in stellar radii). Present-day solar field strength (thick solid line) is compared with that of ages: $\log _{10} t=$ 5.5 (dotted line), 6.0 (dot-dashed line), and 6.5 (dashed line). Also shown is an ideal dipolar field having the same strength at the photosphere as the other cases (thin solid line).

et al. (2007), and the frequency scale was shifted up or down with the photospheric value of the acoustic cutoff frequency, $\omega_{\mathrm{ac}}=c_{s} / 2 H_{*}$, which evolves over time.

2. The photospheric Alfvén wave amplitude $v_{\perp *}$ is specified instead of the upward energy flux $F_{\mathrm{A}}$ because the latter depends on the cancellation between upward and downward propagating waves that is determined as a part of the self-consistent solution. As discussed in $\S 3.4$, the solar value of $0.255 \mathrm{~km} \mathrm{~s}^{-1}$ was supplemented by the accretion-driven wave component. The shape of the Alfvén wave frequency spectrum was kept fixed at the solar model because it is unclear how it should scale with varying stellar properties.

3. The photospheric Alfuén wave correlation length $\ell_{\perp *}$ sets the scale of the turbulent heating rate $Q_{\mathrm{A}}$ (eq. [29]). Once this parameter is set, the value of $\ell_{\perp}$ at larger distances is determined by the assumed proportionality with $A^{1 / 2}$. The solar value of $75 \mathrm{~km}$ (Cranmer et al. 2007) was evolved in proportion with changes in the photospheric scale height $H_{*}$. The justification for this is that the horizontal scale of convective granulation is believed to be set by the scale height (e.g., Robinson et al. 2004), and the turbulent mixing scale is probably related closely to the properties of the granulation.

The numerical relaxation method used by ZEPHYR was discussed by Cranmer et al. (2007). In the absence of explicit specification here, the new T Tauri wind models use the same parameters as given in that paper. However, the new models use a slightly stronger form for the code's iterative undercorrection than did the original solar models. From one iteration to the next, ZEPHYR replaces old values with with a fractional step toward the newly computed values, rather than using the new values themselves. This technique was motivated by globally convergent backtracking methods for finding roots of nonlinear equations (e.g., Dennis \& Schnabel 
1983). The solar models used a constant minimum undercorrection exponent $\epsilon_{0}=0.17$, as defined in equation (65) of Cranmer et al. (2007). The T Tauri models started with this value, but gradually decreased it over time by multiplying $\epsilon_{0}$ by 0.97 after each iteration. The value of $\epsilon_{0}$ was not allowed to be smaller than an absolute minimum of 0.001. This represented an additional kind of "annealing" that helped the parameters reach their time-steady values more rapidly and robustly.

\section{RESULTS}

\subsection{Standard Age Sequence}

A series of 24 ZEPHYR models was created with ages ranging between $\log _{10} t=5.65$ and 9.66. These models all converged to steady-state mass-conserving wind outflow solutions within 250 numerical iterations. The relative errors in the energy equation, averaged over the radial grid, were of order $1 \%$ for the final converged models. For ages younger than $\log _{10} t=5.65$ (i.e., $0.45 \mathrm{Myr}$ ), it was found that time-steady solutions to equations (23)-(25) do not exist, and the best we can do is to estimate the mass flux that is driven up through the Parker critical point (see $§ \S 5.2-5.3$ below). Figure 7 presents a summary of various scalar properties for the wind models as a function of age.

The mass loss rate $\dot{M}_{\text {wind }}$, shown in Figure $7 a$, was calculated by multiplying the mass flux $\rho u$ at the largest grid radius $\left(1200 R_{*}\right.$ for all models) by the full spherical area $4 \pi r^{2}$ at that radius. This is a slight overestimate, since the polar flux tubes only cover a finite fraction of the stellar surface $\left(\delta_{\mathrm{pol}}<1\right)$. However, it is unknown whether these regions expand outward or inward (i.e., to larger or smaller solid angle coverage) with increasing distance from the star. The actual large-scale geometry is likely to depend on how the pressure (gas and magnetic) in the field lines that thread the accretion disk is able to confine or collimate the polar flux tubes.

As the age is decreased, $\dot{M}_{\text {wind }}$ for the time-steady models increases by four orders of magnitude, from the presentday solar value of about $2 \times 10^{-14} M_{\odot} \mathrm{yr}^{-1}$ up to $4 \times 10^{-10}$ $M_{\odot} \mathrm{yr}^{-1}$ at the youngest modeled age of $0.45 \mathrm{Myr}$. Note that these values exceed the mass loss rates predicted by the empirical scaling relation of Reimers $(1975,1977)$ at all ages (i.e., using $\dot{M}_{\text {wind }} \propto L_{*} R_{*} / M_{*}$ and normalizing to the present-day mass loss rate), but for $t \gtrsim 100 \mathrm{Myr}$ there is rough agreement. The stellar wind velocity at the largest radius is denoted as an "asymptotic" or terminal speed $u_{\infty}$ and is shown in Figure $7 b$. The wind speed remains roughly constant for most of the later phase of the evolution (with $u_{\infty} \approx V_{\mathrm{esc}}$ ), but it drops precipitously in the youngest models.

The dominant physical processes that drive the evolving stellar winds are revealed when examining the maximum temperatures in the models, as shown in Figure $7 c$. The older $(t \gtrsim 1.75 \mathrm{Myr})$ models with high wind speeds and solar-like mass loss rates have hot coronae, with peak temperatures between $10^{6}$ and $2 \times 10^{6} \mathrm{~K}$. The younger models undergo a rapid drop in temperature, ultimately leading to "extended chromospheres" with peak temperatures around $10^{4} \mathrm{~K}$. This transition occurs because of a well-known thermal instability in the radiative cooling rate $Q_{\text {rad }}$ (e.g., Parker 1953; Field 1965; Rosner et al. 1978; Suzuki 2007). At temperatures below about $10^{5} \mathrm{~K}$, the cooling rate decreases with decreasing $T$, and small temperature perturbations are easily stabilized. However, above $10^{5} \mathrm{~K}, Q_{\text {rad }}$ decreases as $T$ increases, which gives any small increase in temperature an unstable runaway
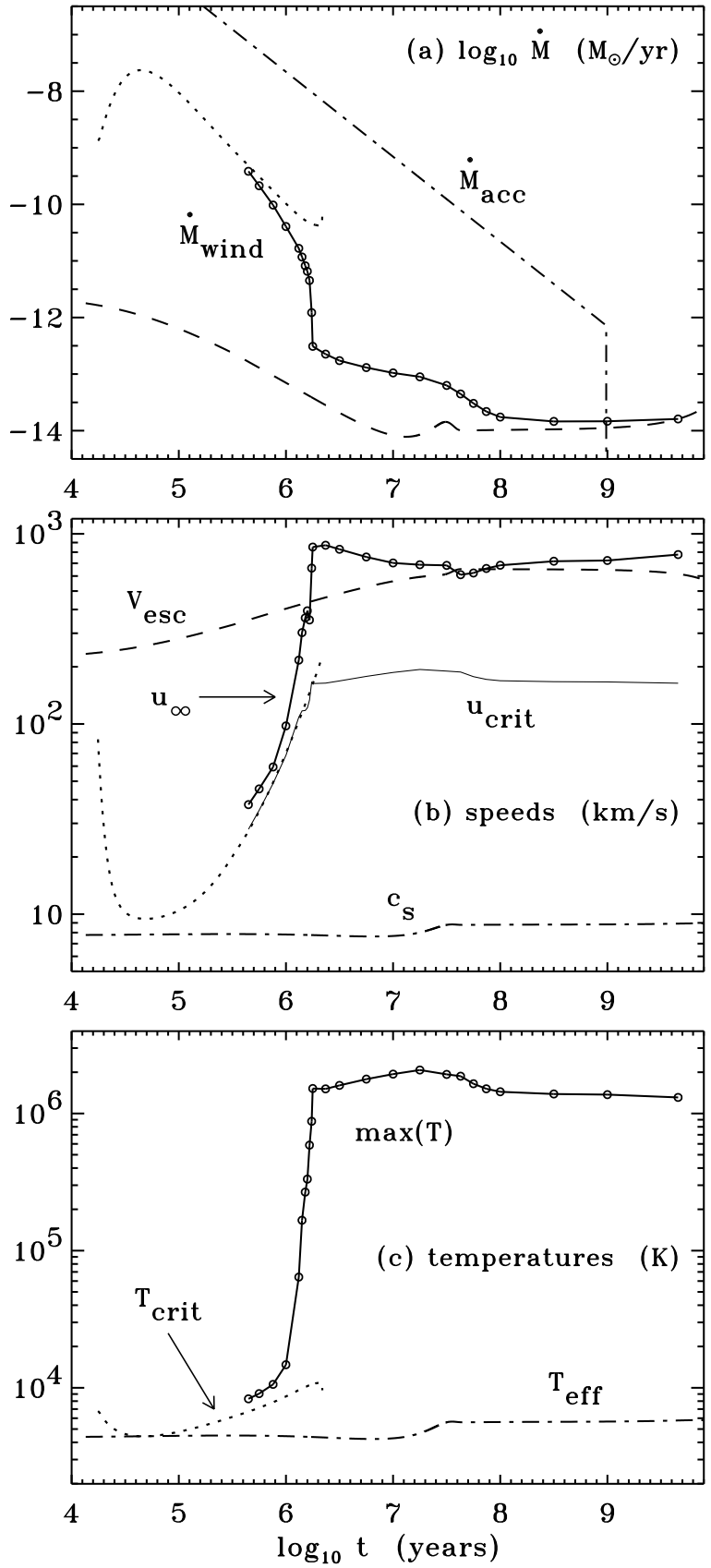

FIG. 7.- Age-dependent model properties. (a) Mass loss rates of timesteady ZEPHYR models (solid line with symbols) compared with the Reimers $(1975,1977)$ relation (dashed line), analytic/cold models of $\$ 5.3$ (dotted line), and adopted accretion rate (dot-dashed line). (b) Terminal wind speed for ZEPHYR models (thick solid line with symbols), surface escape speed (dashed line), photospheric sound speed (dot-dashed line), and wind speed at the wave-modified critical point for ZEPHYR models (thin solid line) and analytic/cold models (dotted line). (c) Peak temperatures of ZEPHYR models (solid line with symbols), temperatures at critical point for analytic/cold models (dotted line), and stellar $T_{\text {eff }}$ (dot-dashed line).

toward larger values. This is the same instability that helps lead to the sharp transition region between the $10^{4} \mathrm{~K}$ solar chromosphere and the $10^{6} \mathrm{~K}$ corona (see also Hammer 1982; Withbroe 1988; Owocki 2004).

The reason that the young and old models in Figure 7 end up on opposite sides of the thermal instability is that the total rates of energy input (i.e., the wave heating rates $Q_{\mathrm{A}}$ and $Q_{\mathrm{S}}$ ) vary strongly as a function of age. The older models have lower wave amplitudes and thus weaker heating rates, which 
leads to relatively low values of $\dot{M}_{\text {wind }}$. The correspondingly low atmospheric densities in these models give rise to weak radiative cooling (because $Q_{\text {rad }} \propto \rho^{2}$ ) that cannot suppress the coronal heating. The coronal winds are driven by comparable contributions from gas pressure and wave pressure. On the other hand, the younger models have larger wave amplitudes, more energy input (as well as more wave pressure, which expands the density scale height), and thus more massive winds with stronger radiative cooling. These chromospheric winds are driven mainly by wave pressure. Related explanations for cool winds from low-gravity stars have been discussed by, e.g., Antiochos et al. (1986), Rosner et al. (1991, 1995), Willson (2000), Killie (2002), and Suzuki (2007).

The processes that determine the quantitative value of $\dot{M}_{\text {wind }}$ are different on either side of the thermal bifurcation. The mass flux for the older, solar-like models can be explained well by the energy balance between heat conduction, radiative losses, and the upward enthalpy flux. The atmosphere finds the height of the transition region that best matches these sources and sinks of energy in a time-steady way, and the resulting gas pressure at this height sets the mass flux. Various analytic solutions for this balance have been given by Hammer (1982), Withbroe (1988), Leer et al. (1998), and Cranmer (2004). The younger, more massive wind models are found to approach the limit of "cold" wave-driven outflows, where the Alfvén wave pressure replaces the gas pressure as the primary means of canceling out the stellar gravity. In this limit, the analytic approach of Holzer et al. (1983), discussed further in $\S 5.3$, has been shown to provide a relatively simple estimate for the mass loss rate.

Figure 8 illustrates a selection of other parameters of the ZEPHYR models. The heights of the wave-modified critical point (see eq. 32] below) and the Alfvén point (where $u=V_{\mathrm{A}}$ ) are shown in units of the stellar radius. The ratio of the isothermal sound speed $a$ to the wind speed at the critical point is also shown. This ratio is close to unity for the older, less massive models (indicating that gas pressure dominates the wind acceleration) and is less than 1 for the younger (more wave-driven) models. Note also that the older "coronal" models have critical points in the sub-Alfvénic wind $\left(u<V_{\mathrm{A}}\right)$ and the younger "extended chromosphere" models have larger critical radii that are in the super-Alfvénic $\left(u>V_{\mathrm{A}}\right)$ part of the wind. Also plotted in Figure $8 a$ is the so-called wind luminosity $L_{\text {wind }}$, which we estimate from the sum of the energy required to lift the wind out of the gravitational potential and the remaining kinetic energy of the flow, i.e.,

$$
L_{\text {wind }}=\dot{M}_{\text {wind }}\left(\frac{G M_{*}}{R_{*}}+\frac{u^{2}}{2}\right)
$$

(e.g., Clayton 1966). This expression ignores thermal energy, ionization energy, magnetic energy, and waves, which are expected to be small contributors at and above the critical point. Curves for the ratio $L_{\text {wind }} / L_{*}$ are shown both for the wind at its largest height (i.e., using $u_{\infty}$ in eq. [30]) and at the critical point (using $u_{\text {crit }}$ ). The latter is helpful to compare with analytic estimates for younger ages described below-for which nothing above the critical point was computed.

Figure $8 b$ shows the terms that dominate the energy conservation equation (at the critical point) as a function of age. The areas plotted here were computed by normalizing the absolute values of the individual terms in equation (25) by the maximum absolute value for each model and then "stacking" them so that together they fill the region between 0 and 1 . The older coronal models have a three-part balance between
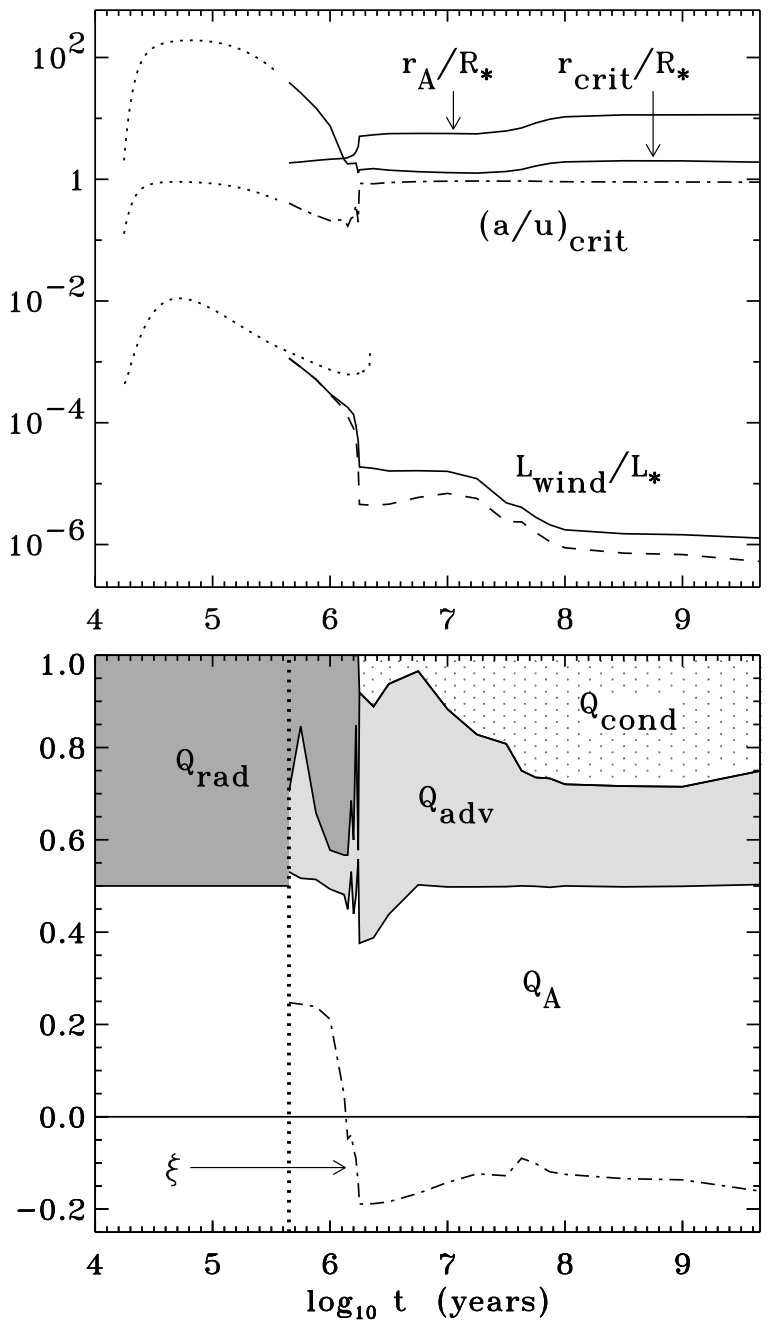

FIG. 8. - Additional age-dependent model properties. (a) Wave-modified critical radii and Alfvén radii (upper solid lines, labeled), ratio of sound speed to wind speed at critical radius (dot-dashed line), and ratio of wind luminosity to photon luminosity, measured at the largest modeled radius (lower solid line) and at the critical point (dashed line). Results for the analytic/cold models are also shown (dotted lines). (b) Areas denote the relative contribution of terms to the energy conservation equation at the critical point; see labels. ( $Q_{\text {adv }}$ denotes the advection terms on the left-hand side of equation [25, other terms are defined in the text.) Also plotted is the exponent $\xi$ versus age (dotdashed line).

Alfvén wave heating, heat conduction, and the upward advection of enthalpy due to the terms on the left-hand side of equation (25). For the younger models, radiative losses become important because of the higher densities at the critical point, and heat conduction disappears because the temperatures are so low.

Figure 9 displays the radial dependence of wind speed, temperature, and density for three selected ages: (1) the presentday polar outflow of the Sun, (2) a younger, but still low- $\dot{M}_{\text {wind }}$ coronal wind, and (3) an even younger model that has made the transition to a higher mass loss rate and an extended chromosphere. The plotted hydrogen number density $n_{\mathrm{H}}$ is that of all hydrogen nuclei (both neutral and ionized). Note that the model with the lowest temperature would be expected to have a small density scale height (eq. [1]) and thus a rapid radial decline in $n_{\mathrm{H}}$. However, Figure $9 c$ shows the opposite to be the case: the coolest model has the largest effective density scale height of the three because of a much larger contribution from wave pressure. 

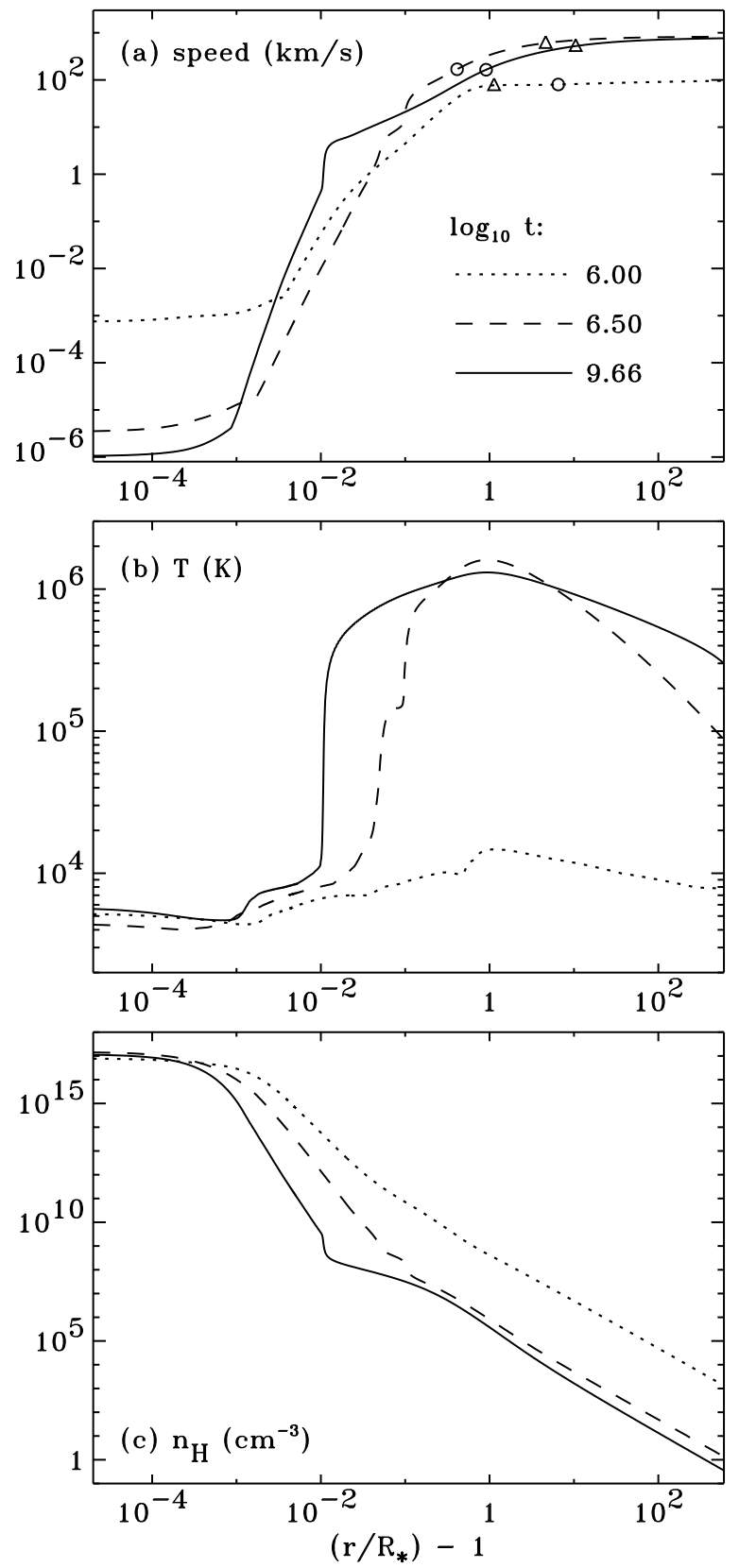

FIG. 9.- Radial dependence of wind parameters for three selected ZEPHYR models: $(a)$ wind outflow velocity, $(b)$ temperature, and $(c)$ total hydrogen number density. In all panels, models are shown for ages $\log _{10} t=6.0$ (dotted lines), 6.5 (dashed lines), and 9.66 (solid lines). Wavemodified critical radii (circles) and Alfvén radii (triangles) are also shown.

There are two additional points of comparison with other work that should be made in the light of the main results shown in Figures 7-9.

1. There is a relatively flat age dependence of the predicted mass loss rate for the post-T Tauri phase (i.e., zero-age main sequence [ZAMS] stars with $t \gtrsim 50$ Myr). This stands in contrast to the observationally inferred power-law decrease for these stages, which is approximately $\dot{M}_{\text {wind }} \propto t^{-2}$ (e.g., Wood et al. 2002, 2005). Note, however, that the internal (convection-related) source of MHD wave energy at the photosphere was assumed in the models to be set at the present-day solar level and not to vary with age. As described in $\S 6$, the inclusion of rotation-dependent convection may give rise to a stronger variation in the turbulent fluctuation amplitude with age, and thus also a more pronounced age dependence of the rates of coronal heating and mass loss.

2. Suzuki (2007) modeled the extended atmospheres and winds of low-gravity cool stars, and found that the onset of thermal instability gave rise to large amounts of time variability. These models often showed dense and cool shells that coexist with spontaneously produced hot and tenuous "bubbles." Suzuki (2007) noted that this dynamical instability began to occur once the escape velocity $V_{\text {esc }}$ (measured in the wind acceleration region) dropped to the point of being of the same order of magnitude as the sound speed corresponding to the thermal instability temperature of $\sim 10^{5} \mathrm{~K}$. It is unclear, though, whether this variability is triggered only for relatively moderate rates of turbulent energy input, like the amplitudes derived by Suzuki (2007) from non-rotating convection models. The larger amplitudes used in the present models of accretion-driven waves may be sufficient to drive the winds much more robustly past the thermal instability and into time-steady extended chromospheres (see also Killie 2002).

\subsection{Disappearance of Time-Steady Solutions}

The ZEPHYR code could not find time-independent wind solutions for ages younger than about $0.45 \mathrm{Myr}$ (i.e., for accretion rates larger than about $\left.7 \times 10^{-8} M_{\odot} \mathrm{yr}^{-1}\right)$. This was found not to be a numerical effect. Instead, it is an outcome of the requirement that time-steady winds must have a sufficient amount of outward acceleration (either due to gas pressure or some other external forcing, like waves) to drive material out of the star's gravitational potential well to a finite coasting speed at infinity. This was realized for polytropic gas-pressure-driven winds very early on (Parker 1963; Holzer \& Axford 1970), such that if the temperature decreases too rapidly with increasing distance (i.e., with decreasing density) the wind would become "stalled" and not have a time-steady solution.

To illustrate how this effect occurs for the youngest, wavedriven models, let us write and analyze an approximate equation of momentum conservation. For simplicity, the wind temperature $T$ is assumed to be constant, and the radii of interest are far enough from the star such that the flux-tube expansion can be assumed to be spherical, with $A \propto r^{2}$. Also, for these models the acoustic wave pressure can be ignored (since compressive waves damp out rather low in the atmosphere), and the radial behavior of the Alfvén wave amplitude can be modeled roughly in the dissipationless limit. Thus, one can follow Jacques (1977) and write the momentum equation as a modified critical point equation

$$
\left(u-\frac{u_{\mathrm{crit}}^{2}}{u}\right) \frac{d u}{d r}=-\frac{G M_{*}}{r^{2}}+\frac{2 u_{\mathrm{crit}}^{2}}{r} .
$$

At the wave-modified critical point $\left(r_{\text {crit }}\right)$, the wind speed $u$ equals the critical speed $u_{\text {crit }}$, which is defined as

$$
u_{\text {crit }}^{2}=a^{2}+\frac{v_{\perp}^{2}}{4}\left(\frac{1+3 M_{\mathrm{A}}}{1+M_{\mathrm{A}}}\right)
$$

where the squared isothermal sound speed is $a^{2}=(1+$ $x) k_{\mathrm{B}} T / m_{\mathrm{H}}$, and the bulk-flow Alfvén Mach number $M_{\mathrm{A}}=$ 
$u / V_{\mathrm{A}}$. A more general version of equation (31) that also contains damping and acoustic wave pressure is given in, e.g., $§ 6$ of Cranmer et al. (2007).

The above expressions show how the outward pressure, which balances gravity at the critical point, can be dominated either by $a^{2}$ (gas pressure) or by a term proportional to $v_{\perp}^{2}$ (wave pressure). For gas pressure that can be described as a polytrope (i.e., $a^{2} \propto \rho^{\gamma-1}$ ), the polytropic index $\gamma$ at the critical point must be smaller than 1.5 in order for there to be a time-steady acceleration from the critical point to infinity (Parker 1963; see also Velli 2001; Owocki 2004). Larger polytropic indices $\gamma \geq 1.5$ imply that $a^{2}$ drops too rapidly with increasing distance to provide sufficient acceleration for a parcel accelerated through the critical point to escape to infinity.

For winds dominated by wave pressure, it is possible to use the equation of wave action conservation (eq. [36]) to examine the density dependence of the wave amplitude in a similar way as above (e.g., Jacques 1977; Heinemann \& Olbert 1980; Cranmer \& van Ballegooijen 2005). The exponent $\xi$ in the scaling relation $v_{\perp} \propto \rho^{\xi}$ is known to be a slowly varying function of distance. Close to the star, where $u \ll V_{\mathrm{A}}$, the exponent $\xi \approx-0.25$. In the vicinity of the Alfvén point $\left(u \approx V_{\mathrm{A}}\right)$, $\xi$ increases to zero and $v_{\perp}(r)$ has a local maximum. Far from the star, where $u \gg V_{\mathrm{A}}$, the exponent $\xi$ grows to an asymptotically large value of +0.25 . The dimensionless quantity in parentheses in equation (32) varies only between 1 and 3 over the full range of distances, and can be assumed to be roughly constant compared to the density dependence of $v_{\perp}$.

Thus, by comparing the polytropic, gas-pressure dominated expression for $u_{\text {crit }}^{2}$ to the wave-pressure dominated version, it becomes possible to write an effective polytropic index for the latter case as $\gamma_{\mathrm{eff}}=2 \xi+1$. The unstable region of $\gamma \geq 1.5$ corresponds to $\xi \geq 0.25$, and this value is reached at the critical point only when $r_{\text {crit }}$ is well into the super-Alfvénic part of the wind. Indeed, this corresponds to the youngest models shown in Figures 7-9. As $\dot{M}_{\text {wind }}$ increases (with decreasing age), the density in the wind increases, and this leads to a sharp decline in the value of $V_{\mathrm{A}}$ at the critical point. (Just over the span of ages going from $\log _{10} t=6.25$ to $6.0, V_{\mathrm{A}}$ at the critical point drops by a factor of 150.) Figure $8 b$ shows $\xi$ versus age for the ZEPHYR models, where this exponent was first computed for all heights via

$$
\xi=\frac{\partial\left(\ln v_{\perp}\right) / \partial r}{\partial(\ln \rho) / \partial r}
$$

and the value shown is that interpolated to the location of the wave-modified critical point. It is clear that $\xi$ approaches the unstable limiting value of 0.25 just at the point where the time-steady solutions disappear.

What happens to a stellar outflow when "too much" mass is driven up past the critical point to maintain a time-steady wind? An isolated parcel of gas with an outflow speed $u=u_{\text {crit }}$ at the critical point would be decelerated to stagnation at some height above the critical point, and it would want to fall back down towards the star. In reality, this parcel would collide with other parcels that are still accelerating, and a stochastic collection of shocked clumps is likely to result. Interactions between these parcels may result in an extra degree of collisional heating that could act as an extended source of gas pressure to help maintain a mean net outward flow. Situations similar to this have been suggested to occur in the outflows of both pulsating cool stars (e.g., Bowen 1988; Willson 2000; Struck et al. 2004) and luminous blue variables (Owocki \& van Marle 2008). The models presented here suggest that the most massive stellar winds $\left(\dot{M}_{\text {wind }} \gtrsim 10^{-9} M_{\odot} \mathrm{yr}^{-1}\right)$ of young $\mathrm{T}$ Tauri stars may exist in a similar kind of superposition of outflowing and inflowing shells.

\subsection{Analytic Estimate of Wave-Driven Mass Loss}

For the youngest $\mathrm{T}$ Tauri models with (seemingly) no steady state, it is possible to use an analytic technique to estimate how much mass gets accelerated up to the wave-modified critical point. As described above, it is not certain whether all of this mass can be accelerated to infinity. However, the ability to determine a mass flux that applies to the finite region between the stellar surface and the critical radius may be sufficient to predict many observed mass loss diagnostics. Figure $8 a$ showed that $r_{\text {crit }} \gg R_{*}$ for these young ages, and thus the "subcritical volume" is relatively large.

The modified critical point equation given above for the limiting case of dissipationless Alfvén waves has been studied for several decades (e.g., Jacques 1977; Hartmann \& MacGregor 1980; DeCampli 1981; Holzer et al. 1983; Wang \& Sheeley 1991). Equation (31) can be solved for the critical point radius by setting the right-hand side to zero, to obtain

$$
r_{\text {crit }}=\frac{G M_{*}}{2 u_{\text {crit }}^{2}}, u_{\text {crit }}^{2}=a^{2}+\frac{3 v_{\perp \text { crit }}^{2}}{4}
$$

where it is assumed that $u \gg V_{\mathrm{A}}$ (or $M_{\mathrm{A}} \gg 1$ ) at the critical point, which applies in the youngest T Tauri models. In order to compute a value for the critical point radius, however, we would have to know the value of $v_{\perp \text { crit }}$, the Alfvén wave amplitude at the critical point. No straightforward (a priori) method was found to predict $v_{\perp \text { crit }}$ from the other known quantities derived in $\S 3$. Thus, we relied on an empirical fitting relation that was produced from the 6 youngest ZEPHYR models (with $0.45 \leq t \leq 1.4 \mathrm{Myr}$ ),

$$
\frac{v_{\perp \text { crit }}}{18.9 \mathrm{~km} \mathrm{~s}^{-1}}=\left(\frac{v_{\perp *}}{10 \mathrm{~km} \mathrm{~s}^{-1}}\right)^{-2.37}
$$

where $v_{\perp *}$ is the Alfvén wave amplitude at the photosphere. The fit is good to $5 \%$ accuracy for the 6 youngest numerical models, but it is not known whether this level of accuracy persists when it is extrapolated to ages younger than $\sim 0.45$ Myr.

The assumption that the Alfvén waves are dissipationless allows the conservation of wave action to be used, i.e.,

$$
\frac{\rho v_{\perp}^{2}\left(u+V_{\mathrm{A}}\right)^{2} A}{V_{\mathrm{A}}}=\text { constant } .
$$

This can be simplified by noting that, for the youngest $\mathrm{T}$ Tauri models, the photosphere always exhibits $u \ll V_{\mathrm{A}}$, and the wave-modified critical point always exhibits $u \gg V_{\mathrm{A}}$. Thus, with these assumptions, equation (36) can be applied at these two heights to obtain

$$
\rho_{*} v_{\perp *}^{2} V_{\mathrm{A} *} A_{*} \approx \frac{\rho_{\text {crit }} v_{\perp \text { crit }}^{2} u_{\text {crit }}^{2} A_{\text {crit }}}{V_{\text {A crit }}} .
$$

The main unknown is the density $\rho_{\text {crit }}$ at the critical point, which can be solved in terms of the other known quantities,

$$
\left(\frac{\rho_{\text {crit }}}{\rho_{*}}\right)^{3 / 2} \approx \frac{B_{\text {crit }}^{2}}{4 \pi \rho_{*} u_{\text {crit }}^{2}}\left(\frac{v_{\perp *}}{v_{\perp \text { crit }}}\right)^{2}
$$

and thus the mass flux is determined uniquely at the critical point (Holzer et al. 1983). The mass loss rate is $\dot{M}_{\text {wind }}=$ $\rho_{\text {crit }} u_{\text {crit }} A_{\text {crit }}$, where the critical flux-tube area $A_{\text {crit }}$ is normalized such that as $r \rightarrow \infty, A \rightarrow 4 \pi r^{2}$. 
Although the above provides a straightforward algorithm for estimating $\dot{M}_{\text {wind }}$ for a wave-driven wind, there is one other unspecified quantity: the isothermal sound speed $a$ at the critical point. A completely "cold" wave-driven model would set $a=0$, but it was found that the extended chromospheres in the youngest ZEPHYR models do contribute some gas pressure to the overall acceleration. The present set of approximate models began with an initial guess for the critical point temperature $\left(T_{\text {crit }} \approx 10^{4} \mathrm{~K}\right)$ and then iterated to find a more consistent value. The iteration process involved alternating between solving for $\dot{M}_{\text {wind }}$ at the critical point (as described above) and recomputing $T_{\text {crit }}$ by assuming a balance between Alfvén-wave turbulent heating and radiative cooling at the critical point. The turbulent heating rate $Q_{\mathrm{A}}$ was computed as in equation (29), and the cooling rate $Q_{\text {rad }}$ was assumed to remain proportional to its optically thin limit $\rho^{2} \Lambda(T)$ (i.e., ignoring the $\tau_{\mathrm{hk}}$ correction factor described in $\S 4.1$ ). The temperature at which this balance occurred was found by inverting the tabulated radiative loss function $\Lambda(T)$, shown in Figure 1 of Cranmer et al. (2007). For each age-between $13.5 \mathrm{kyr}$ and $0.45 \mathrm{Myr}$ - this process was run for 20 iterations, but in all cases it converged rapidly within the first 5 iterations.

The results of this iterative estimation of $\dot{M}_{\text {wind }}, T_{\text {crit }}$, and $u_{\text {crit }}$ are shown by the dotted curves in Figures 7 and 8 . The maximum mass loss rate of $2.4 \times 10^{-8} M_{\odot} \mathrm{yr}^{-1}$ occurs at an age of about $40 \mathrm{kyr}$. At the youngest ages, the mass loss rate declines because the accretion-driven wave power also declines (see Fig. 5). The most massive wind corresponds to the minimum value of the temperature at the critical point, which is indistinguishable from the stellar $T_{\mathrm{eff}}$ at that age. At the oldest ages for which these solutions were attempted $(t \gtrsim 1.5$ $\mathrm{Myr}$ ), the various approximations made above no longer hold (e.g., the critical point is no longer super-Alfvénic) and the agreement between the analytic estimates and the ZEPHYR models breaks down.

\subsection{Varying the Accretion Rate}

As shown in Figure 3 above, the measured values for $\dot{M}_{\text {acc }}$ exhibit a wide spread around the mean relation that was used to derive the properties of accretion-driven waves. In order to explore how variability in the accretion rate affects the resulting stellar wind, a set of additional ZEPHYR models was constructed with a factor of two decrease and increase in $\dot{M}_{\text {acc }}$ in comparison to equation (3). These models were constructed mainly for ages around the thermal bifurcation at $\log _{10} t \approx 6.25$. It was found that the older "hot corona" models were insensitive to variations in the accretion rate, even at ages where the accretion-driven component to $v_{\perp *}$ exceeded the internal convective component.

Figure 10 compares the mass loss rate and maximum wind temperature for the three sets of ZEPHYR models: the original standard models, ones with half of the accretion rate given by equation (3), and ones with double that rate. The specific age at which the thermal bifurcation occurs changes by only a small amount over the range of modeled accretion rates. (Indeed, the standard model and the double- $\dot{M}_{\text {acc }}$ model undergo thermal bifurcation at nearly the exact same age.) The youngest and coolest models show a greater degree of responsiveness to the varying accretion rate than do the older hot models. The younger models with higher accretion rates have larger photospheric MHD wave amplitudes, and thus they give rise to larger mass loss rates and cooler extended
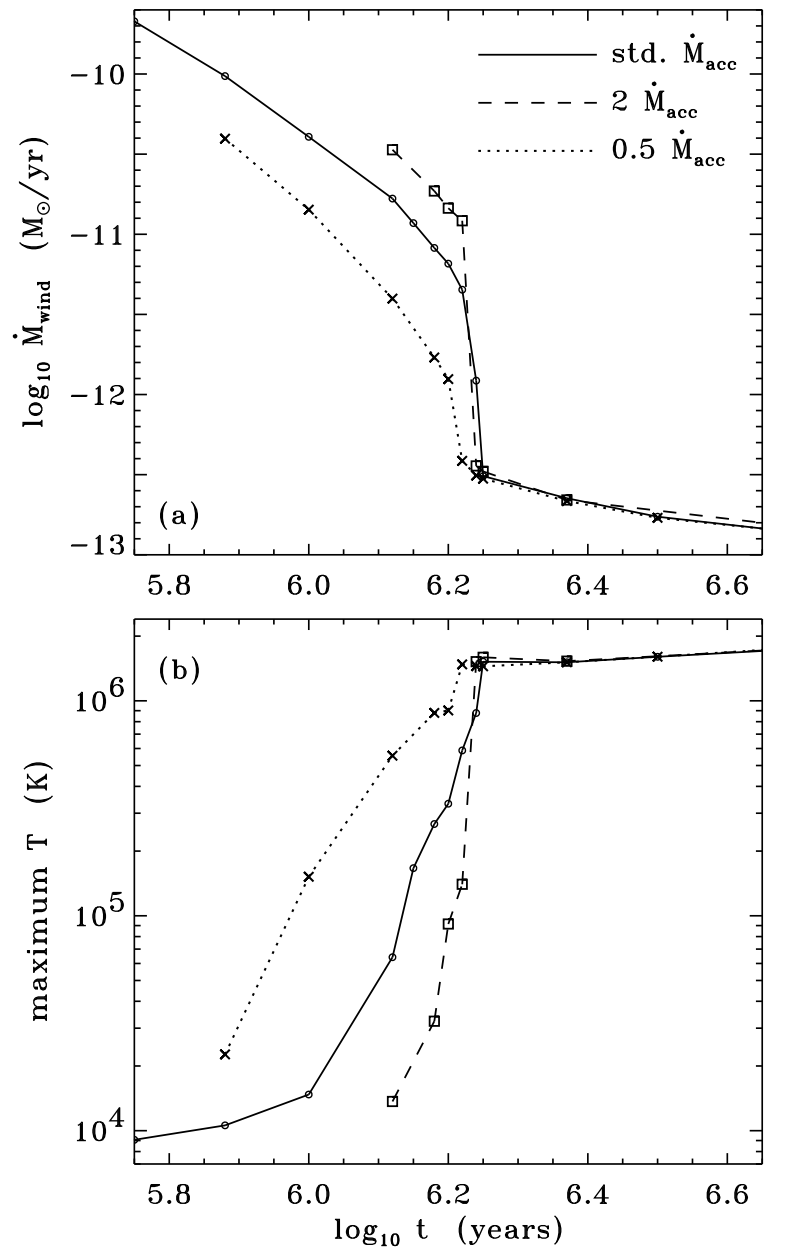

FIG. 10.- Age-dependent mass loss rates $(a)$ and peak wind temperatures $(b)$ for ZEPHYR models produced with the standard accretion rate from eq. (3) (solid lines), and accretion rates that are double (dashed lines) and half (dotted lines) the standard values. Individual ZEPHYR models are denoted by symbols.

\section{chromospheres.}

Figure 11 shows how both $\dot{M}_{\text {acc }}$ and $\dot{M}_{\text {wind }}$ vary as a function of the photospheric Alfvén wave amplitude $v_{\perp *}$. This latter quantity is a key intermediary between the mid-latitude accretion and the polar mass loss, and thus it is instructive to see how both mass flux quantities scale with its evolving magnitude. The three sets of ZEPHYR models from Figure 10 are also shown in Figure 11, and it is noteworthy that there is not a simple one-to-one correspondence between $\dot{M}_{\text {acc }}$ and $v_{\perp *}$. The small spread arises because the fundamental stellar parameters (e.g., $R_{*}$ and $\rho_{*}$ ) are different at the three ages that correspond to the same value of $\dot{M}_{\text {acc }}$ in the three models. Thus, it is not surprising that $\dot{M}_{\text {wind }}$ is not a "universal" function of the wave amplitude either.

Figure 12 plots the key "wind efficiency" ratio $\dot{M}_{\text {wind }} / \dot{M}_{\text {acc }}$ as a function of age for the three sets of ZEPHYR models and the analytic estimates derived in $\S 5.3$. Note that, in contrast to Figure 10, when plotted as a ratio, the models on the young/cool side of the thermal bifurcation all seem to collapse onto a single curve, while the older/hotter models separate (based on differing accretion rates in the denominator).

For both the youngest ages $(t \lesssim 0.5 \mathrm{Myr})$ and for the specific case of TW Hya, the model values shown in Figure 12 seem to agree reasonably well with the observationally determined ratios that are also shown in Figure $3 b$. The mod- 


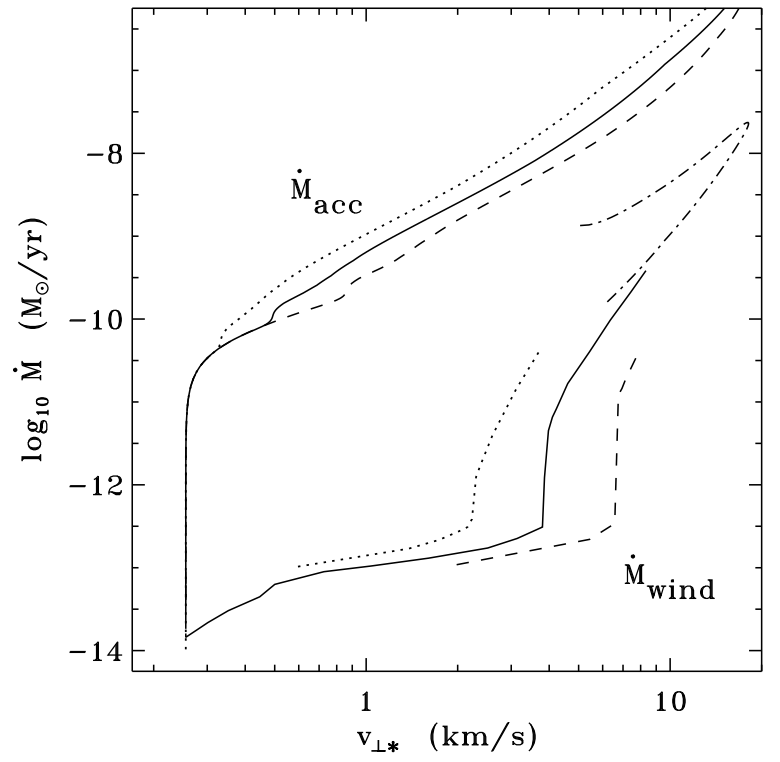

FIG. 11. - Mass accretion and mass loss rates for the 3 sets of ZEPHYR models shown in Figure 10 (with the same line styles) shown versus the photospheric Alfvén wave amplitude $v_{\perp *}$. Mass loss rates for the analytic/cold models of $\S 5.3$ are also shown for the standard accretion rate case (dotdashed line).

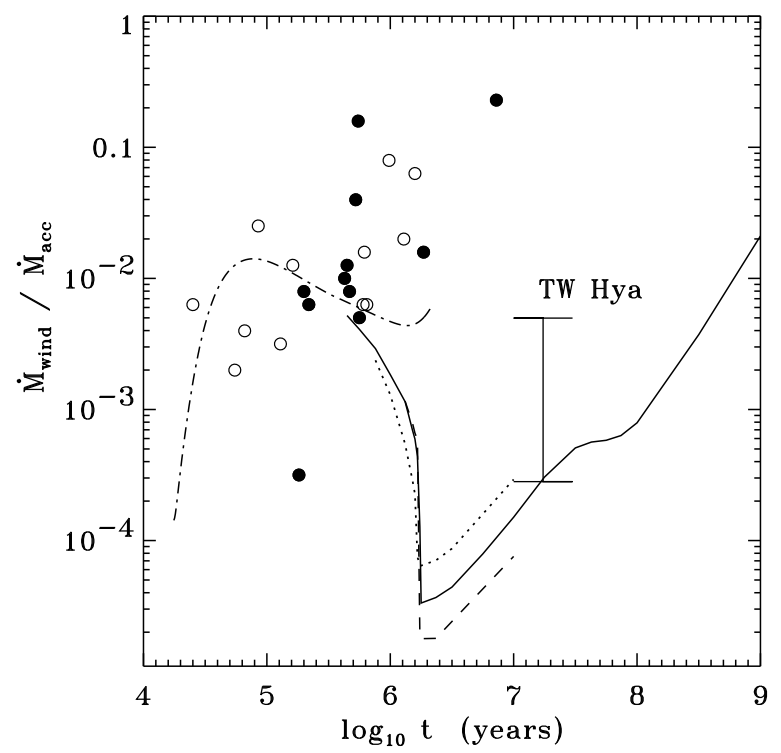

FIG. 12.- Ratio of mass loss rate to mass accretion rate for measured $\mathrm{T}$ Tauri stars (symbols as in Fig. 3) and for the ZEPHYR and analytic models (line styles as in Figs. 10 and 11).

els having ages between 0.5 and $10 \mathrm{Myr}$ clearly fall well below the measured mass loss rates. However, even the limited agreement with the data is somewhat surprising, since these measured values come from the [O I] $\lambda 6300$ forbidden line diagnostic that is widely believed to sample the much largerscale disk wind (Hartigan et al. 1995). It is thus possible that stellar winds may contribute to observational signatures that previously have been assumed to probe only the (disk-related) bipolar jets.

\subsection{X-Ray Emission}

Many aspects of the dynamics and energetics of young stars and their environments are revealed by high-energy measurements such as X-ray emission (e.g., Feigelson \& Montmerle 1999). It is thus worthwhile to determine the level of X-ray

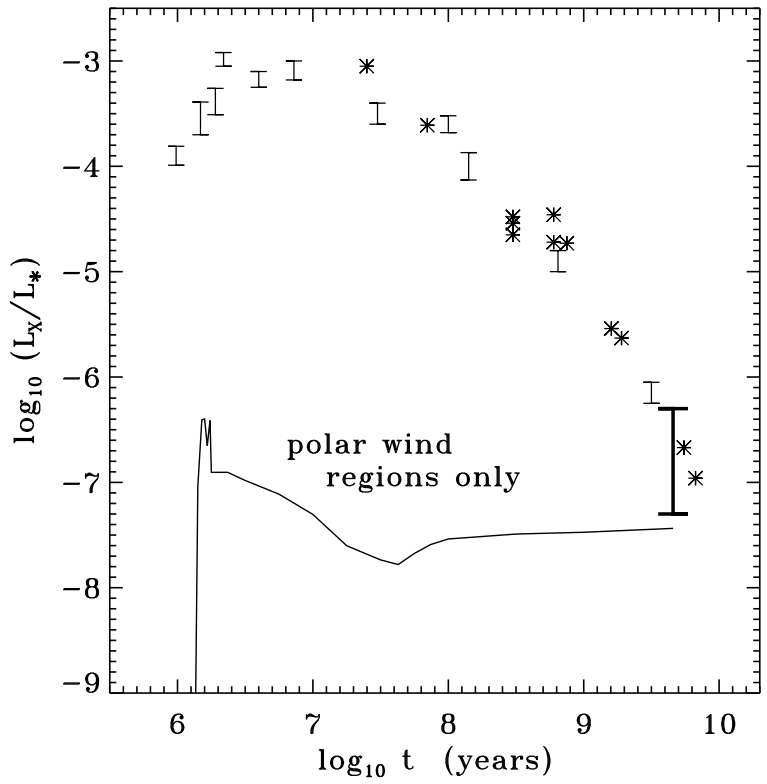

FIG. 13.- Ratio of X-ray luminosity to total bolometric luminosity for the standard run of ZEPHYR models (solid line), and for observations of solartype stars (asterisks), nearby clusters (thin error bars), and the Sun (thick error bar); see text for details.

flux that the modeled polar winds are expected to generate. This has been done in an approximate way to produce orderof-magnitude estimates, and should be followed up by more exact calculations in future work.

The optically thin radiative loss rate $Q_{\text {rad }}$ described in $\S 4.1$ was used as a starting point to "count up" the total number of photons generated by each radial grid zone in the ZEPHYR models. (Finite optical depth effects in $Q_{\text {rad }}$ were ignored here because they contribute mainly to temperatures too low to affect X-ray fluxes.) This rate depends on the plasma temperature $T$, the density $\rho$, and the hydrogen ionization fraction $x$. The total radiative loss rate is multiplied by a fraction $F$ that gives only those photons that would be observable as X-rays. This fraction is estimated for each radial grid zone as

$$
F=\frac{\int d \lambda B_{\lambda}(T) S(\lambda)}{\int d \lambda B_{\lambda}(T)}
$$

where $B_{\lambda}(T)$ is the Planck blackbody function and $S(\lambda)$ is an X-ray sensitivity function, for which we use that of the ROSAT Position Sensitive Proportional Counter (PSPC) instrument, as specified by Judge et al. (2003). The function $S(\lambda)$ is nonzero between about 0.1 and $2.4 \mathrm{keV}$, with a minimum around $0.3 \mathrm{keV}$ that separates the hard and soft bands. The integration in the denominator is taken over all wavelengths. The use of the blackbody function, rather than a true optically thin emissivity, was validated by comparison with wavelength-limited X-ray radiative loss rates given by Raymond et al. (1976). Fractions of emissivity (relative to the total loss rate) in specific X-ray wavebands were computed for $T=0.5,1,2$, and $5 \mathrm{MK}$ and compared with the plots of Raymond et al. (1976). The agreement between the models and the published curves was always better than a factor of two. ${ }^{5}$

${ }^{5}$ Although this is obviously not accurate enough for quantitative comparisons with specific observations, it allows the correct order of magnitude of the X-ray emission to be estimated; see Figure 13. Also, the factor-of-two validation should be taken in the context of the factor of $\sim 1000$ variation in these fractions over the modeled coronal temperatures. 
Figure 13 shows the simulated ratio of X-ray luminosity $L_{\mathrm{X}}$ to the bolometric luminosity $L_{*}$ for the modeled wind regions. For each ZEPHYR model, the radiative losses were integrated over an assumed spherical volume for the stellar wind (i.e., the same assumption used to compute $\dot{M}_{\text {wind }}$; see $\S 5.1$ ) to produce $L_{\mathrm{X}}$. No absorption of X-rays was applied. Figure 13 also shows a collection of observed X-ray luminosity ratios for individual solar-type stars (from Güdel et al. 1998; GarcíaAlvarez et al. 2005) and clusters of various ages (Flaccomio et al. 2003b; Preibisch \& Feigelson 2005; Jeffries et al. 2006). For the latter, the error bars indicate the $\pm 1 \sigma$ spread about the mean values reported in these papers. The range of values for the present-day Sun is taken from Judge et al. (2003).

It is clear from Figure 13 that the modeled polar wind regions do not produce anywhere near enough X-rays to explain the observations of young stars. For the present-day Sun, the computed value slightly underestimates the lower limit of the observed range of X-ray luminosities. This latter prediction does make some sense, since the X-ray emission is essentially computed for the dark "polar coronal holes." On the Sun, these never occupy more than about $20 \%$ of the surface. Most notably, though, there is no strong power-law decrease in $L_{\mathrm{X}} / L_{*}$ for young ZAMS stars-just as there is no power-law decrease in $\dot{M}_{\text {wind }}$-because there has been no attempt to model the rotation-age-activity relationship (Skumanich 1972; Noyes et al. 1984). The ZEPHYR models neglect the closed-field coronae of these stars (both inside and outside the polar-cap regions) that are likely to dominate the X-ray emission, like they do for the Sun (e.g., Schwadron et al. 2006)

It is somewhat interesting that the ZEPHYR models undergo the thermal bifurcation to extended chromospheres (and thus disappear from Figure 13 because of the lack of X-ray emitting temperatures) at about the same age where there seems to exist a slight decline in the X-ray emission of young $\mathrm{T}$ Tauri stars. Although there is some evidence for such a deficit of X-rays in classical T Tauri stars, with respect to the older class of weak-lined T Tauri stars (e.g., Flaccomio et al. 2003a,b; Telleschi et al. 2007), the existence of a distinct peak in X-ray emission at intermediate ages has not been proven rigorously. If the polar wind regions indeed produce negligible X-ray emission compared to the closed-field regions, we note that the polar cap area $\delta_{\text {pol }}$ grows largest for the youngest T Tauri stars (see Fig. 4). Thus, the cooler wind material may occupy a significantly larger volume than the hotter (closedloop) coronal plasma at the youngest ages. This may be partly responsible for the observed $\mathrm{X}$-ray trends.

\section{DISCUSSION AND CONCLUSIONS}

The primary aim of this paper has been to show how accretion-driven waves on the surfaces of $\mathrm{T}$ Tauri stars may help contribute to the strong rates of atmospheric heating and large mass loss rates inferred for these stars. The ZEPHYR code, which was originally developed to model the solar corona and solar wind (Cranmer et al. 2007), has been applied to the T Tauri stellar wind problem. A key aspect of the models presented above is that the only true free parameters are: (1) the properties of MHD waves injected at the photospheric lower boundary, and (2) the background magnetic geometry. Everything else (e.g., the radial dependence of the rates of chromospheric and coronal heating, the temperature structure of the atmosphere, and the wind speeds and mass fluxes) emerges naturally from the modeling process.

For solar-mass T Tauri stars, time-steady stellar winds were found to be supportable for all ages older than about $0.45 \mathrm{Myr}$, with accretion rates less than $7 \times 10^{-8} M_{\odot} \mathrm{yr}^{-1}$ driving mass loss rates less than $4 \times 10^{-10} M_{\odot} \mathrm{yr}^{-1}$. Still younger T Tauri stars (i.e., ages between about $13 \mathrm{kyr}$ and $0.45 \mathrm{Myr}$ ) may exhibit time-variable winds with mass loss rates extending up several more orders of magnitude to $\sim 2 \times 10^{-8} M_{\odot} \mathrm{yr}^{-1}$. The transition between time-steady and variable winds occurs when the critical point of the flow migrates far enough past the Alfvén point (at which the wind speed equals the Alfvén speed) such that the Alfvén wave amplitude begins to decline rapidly with increasing radius. When this happens, the outward wave-pressure acceleration is quickly "choked off;" i.e., parcels of gas that make it past the critical point cannot be accelerated to infinity, and stochastic collisions between upflowing and downflowing parcels must begin to occur.

The maximum wind efficiency ratio $\dot{M}_{\text {wind }} / \dot{M}_{\text {acc }}$ for the $\mathrm{T}$ Tauri models computed here was approximately $1.4 \%$, computed for ages of order $0.1 \mathrm{Myr}$. This is somewhat smaller than the values of order $10 \%$ required by Matt \& Pudritz $(2005,2007,2008)$ to remove enough angular momentum from the young solar system to match present-day conditions. It is also well below the observational ratios derived by, e.g., Hartigan et al. (1995, 2004), which can reach up to $20 \%$ for $\mathrm{T}$ Tauri stars between 1 and $10 \mathrm{Myr}$ old (see Fig. 12). These higher ratios, though, may be the product of both stellar winds and disk winds (possibly even dominated by the disk wind component). Additionally, it is possible that future observational analysis will result in these empirical ratios being revised upward with more accurate (lower) values of $\dot{M}_{\text {acc }}$ (S. Edwards 2008, private communication).

The accretion-driven solutions for $\dot{M}_{\text {wind }}$ depend crucially on the properties of the waves in the polar regions. It is important to note that the calculation of MHD wave properties was based on several assumptions that should be examined in more detail. The relatively low MHD wave efficiency used in equation (17) is an approximation based on the limiting case of waves being far from the impact site. A more realistic model would have to contain additional information about both the nonlinearities of the waves themselves and the vertical atmospheric structure through which the waves propagate. It seems likely that a better treatment of the wave generation would lead to larger wave energies at the poles. On the other hand, the assumption that the waves do not damp significantly between their generation point and the polar wind regions may have led to an assumed wave energy that is too high. It is unclear how strong the waves will be in a model that takes account of all of the above effects. In any case, the ZEPHYR results presented here are the first self-consistent models of $\mathrm{T}$ Tauri stellar winds that produce wind efficiency ratios that even get into the right "ballpark" of the angular momentum requirements.

Additional improvements in the models are needed to make further progress. For example, the effects of stellar rotation should be included, both in the explicit wind dynamics (e.g., "magneto-centrifugal driving," as recently applied by Holzwarth \& Jardine 2007) and in the modified subsurface convective activity that is likely to affect photospheric amplitudes of waves and turbulence. Young and rapidly rotating stars are likely to have qualitatively different convective motions than are evident in standard (nonrotating, mixing-length) models. It is uncertain, though, whether rapid rotation gives rise to larger (Käpylä et al. 2007; Brown et al. 2007; Ballot et al. 2007) or smaller (Chan 2007) convection eddy velocities 
at the latitudes of interest for $\mathrm{T}$ Tauri stellar winds. In any case, rapid rotation can also increase the buoyancy of subsurface magnetic flux elements, leading to a higher rate of flux emergence (Holzwarth 2007). Also, the lower gravities of T Tauri stars may give rise to a larger fraction of the convective velocity reaching the surface as wave energy (e.g., Renzini et al. 1977), or the convection may even penetrate directly into the photosphere (Montalbán et al. 2004).

Future work must involve not only increased physical realism for the models, but also quantitative comparisons with observations. The methodology outlined in this paper should be applied to a set of real stars, rather than to the idealized evolutionary sequence of representative parameters. Measured stellar masses, radii, and $T_{\text {eff }}$ values, as well as accretion rates, magnetic field strengths, and hot spot filling factors $(\delta)$, should be used as constraints on individual calculations for the stellar wind properties. It should also be possible to use measured three-dimensional magnetic fields (e.g., Donati et al. 2007; Jardine et al. 2008) to more accurately map out the patterns of accretion stream footpoints, wave fluxes, and the flux tubes in which stellar winds are accelerated.

This work helps to accomplish the larger goal of understanding the physics responsible for low-mass stellar outflows and the feedback between accretion disks, winds, and stellar magnetic fields. In addition, there are links to more interdisciplinary studies of how stars affect objects in young solar systems. For example, the coronal activity and wind of the young Sun is likely to have created many of the observed abundance and radionuclide patterns in early meteorites (Lee et al. 1998) and possibly affected the Earth's atmospheric chemistry to the point of influencing the development of life (see, e.g., Ribas et al. 2005; Güdel 2007; Cuntz et al. 2008). The identification of key physical processes in young stellar winds is important not only for understanding stellar and planetary evolution, but also for being able to model and predict the present-day impacts of solar variability and "space weather" (e.g., Feynman \& Gabriel 2000; Cole 2003).

I gratefully acknowledge Andrea Dupree, Adriaan van Ballegooijen, Nancy Brickhouse, and Eugene Avrett for many valuable discussions. This work was supported by the National Aeronautics and Space Administration (NASA) under grant NNG04GE77G to the Smithsonian Astrophysical Observatory.

\section{REFERENCES}

Airapetian, V. S., Ofman, L., Robinson, R. D., Carpenter, K., \& Davila, J. 2000, ApJ, 528, 965

Antiochos, S. K., Haisch, B. M., \& Stern, R. A. 1986, ApJ, 307, L55

Appenzeller, I., \& Mundt, R. 1989, A\&A Rev., 1, 291

Argiroffi, C., Maggio, A., \& Peres, G. 2007, A\&A, 465, L5

Aschwanden, M. J. 2006, Physics of the Solar Corona: An Introduction with Problems and Solutions, 2nd ed. (Berlin: Springer)

Avrett, E. H., \& Loeser, R. 2008, ApJS, 175, 229

Balasubramaniam, K. S., Pevtsov, A. A., \& Neidig, D. F. 2007, ApJ, 658, 1372

Ballot, J., Brun, A. S., \& Turck-Chièze, S. 2007, ApJ, 669, 1190

Batalha, C., Batalha, N. M., Alencar, S. H. P., Lopes, D. F., \& Duarte, E. S. 2002, ApJ, 580, 343

Belcher, J. W. 1971, ApJ, 168, 509

Bertout, C. 1989, ARA\&A, 27, 351

Bessolaz, N., Zanni, C., Ferreira, J., Keppens, R., \& Bouvier, J. 2008, A\&A, 478, 155

Bouvier, J., Dougados, C., \& Alencar, S. H. P. 2004, Ap\&SS, 292, 659

Bouvier, J., et al. 1997, A\&A, 318, 495

Bouvier, J., et al. 2003, A\&A, 409, 169

Bouvier, J., et al. 2007, A\&A, 463, 1017

Bowen, G. H. 1988, ApJ, 329, 299

Bretherton, F. P., \& Garrett, C. J. R. 1968, Proc. Roy. Soc. A, 302, 529

Brown, B. P., Browning, M., Brun, A. S., Miesch, M. S., \& Toomre, J. 2007, Astron. Nachr., 328, 1002

Cai, M. J., Shang, H., Lin, H.-H., \& Shu, F. H. 2008, ApJ, 672, 489

Calvet, N. 1997, in IAU Symp. 182, Herbig-Haro Flows and the Birth of Stars, ed. B. Reipurth \& C. Bertout (Dordrecht: Kluwer), 417

Calvet, N., \& Gullbring, E. 1998, ApJ, 509, 802

Calvet, N., \& Hartmann, L. 1992, ApJ, 386, 239

Camenzind, M. 1990, Rev. Mod. Astron., 3, 234

Casse, F., Meliani, Z., \& Sauty, C. 2007, Ap\&SS, 311, 57

Chan, K. L. 2007, Astron. Nachr., 328, 1059

Chevalier, R. A., \& Imamura, J. N. 1982, ApJ, 261, 543

Clayton, D. D. 1966, AJ, 71, 381

Cole, D. G. 2003, Space Sci. Rev., 107, 295

Cranmer, S. R. 2002, Space Sci. Rev., 101, 229

Cranmer, S. R. 2004, Am. J. Phys., 72, 1397

Cranmer, S. R., \& van Ballegooijen, A. A. 2005, ApJS, 156, 265

Cranmer, S. R., van Ballegooijen, A. A., \& Edgar, R. J. 2007, ApJS, 171, 520

Cuntz, M., Gurdemir, L., Guinan, E. F., \& Kurucz, R. L. 2008, in IAU Symp.

249, Exoplanets: Detection, Formation and Dynamics, ed. Y. Sun \& S. Ferraz-Mello (Cambridge: Cambridge Univ. Press), 203

Davidson, K., \& Ostriker, J. P. 1973, ApJ, 179, 585

DeCampli, W. M. 1981, ApJ, 244, 124

Delannée, C., Török, T., Aulanier, G., \& Hochedez, J.-F. 2008, Sol. Phys., 247,123 de Jager, C., \& Nieuwenhuijzen, H. 1987, A\&A, 177, 217

Dennis, J. E., \& Schnabel, R. B. 1983, Numerical Methods for Unconstrained Optimization and Nonlinear Equations (Englewood Cliffs, NJ: PrenticeHall)

Dmitruk, P., \& Matthaeus, W. H. 2003, ApJ, 597, 1097

Dmitruk, P., Matthaeus, W. H., Milano, L. J., Oughton, S., Zank, G. P., \& Mullan, D. J. 2002, ApJ, 575, 571

Dmitruk, P., Milano, L. J., \& Matthaeus, W. H. 2001, ApJ, 548, 482

Donati, J.-F., et al. 2007, MNRAS, 380, 1297

Dupree, A. K., Brickhouse, N. S., Smith, G. H., \& Strader, J. 2005, ApJ, 625, L131

Edwards, S., Fischer, W., Hillenbrand, L., \& Kwan, J. 2006, ApJ, 646, 319

Eggleton, P. P. 1971, MNRAS, 151, 351

Eggleton, P. P. 1972, MNRAS, 156, 361

Eggleton, P. P. 1973, MNRAS, 163, 279

Eggleton, P. P., Faulkner, J., \& Flannery, B. P. 1973, A\&A, 23, 325

Elfimov, A. G., Galvão, R. M. O., Jatenco-Pereira, V., \& Opher, R. 2004, ApJ, 600,292

Elsner, R. F., \& Lamb, F. K. 1977, ApJ, 215, 897

Falceta-Gonçalves, D., Vidotto, A. A., \& Jatenco-Pereira, V. 2006, MNRAS, 368,1145

Feigelson, E. D., \& Montmerle, T. 1999, ARA\&A, 37, 363

Ferreira, J., Dougados, C., \& Cabrit, S. 2006, A\&A, 453, 785

Feynman, J., \& Gabriel, S. B. 2000, J. Geophys. Res., 105, 10543

Field, G. B. 1965, ApJ, 142, 531

Flaccomio, E., Micela, G., \& Sciortino, S. 2003a, A\&A, 397, 611

Flaccomio, E., Micela, G., \& Sciortino, S. 2003b, A\&A, 402, 277

Gail, H. P., \& Sedlmayr, E. 1987, A\&A, 171, 197

García-Alvarez, D., Drake, J. J., Lin, L., et al. 2005, ApJ, 621, 1009

Ghosh, P., \& Lamb, F. K. 1979a, ApJ, 232, 259

Ghosh, P., \& Lamb, F. K. 1979b, ApJ, 234, 296

Giardino, G., Favata, F., Pillitteri, I., Flaccomio, E., Micela, G., \& Sciortino, S. 2007, A\&A, 475, 891

Gómez de Castro, A. I., \& Verdugo, E. 2007, ApJ, 654, L91

Grevesse, N., Asplund, M., \& Sauval, A. J. 2007, Space Sci. Rev., 130, 105

Güdel, M. 2007, Living Rev. Solar Phys., 4, lrsp-2007-3

Güdel, M., Guinan, E. F., \& Skinner, S. L. 1998, in 10th Cambridge Workshop on Cool Stars, Stellar Systems, and the Sun, ASP Conf. Ser. 154,1041

Gullbring, E., Barwig, H., Chen, P. S., Gahm, G. F., \& Bao, M. X. 1996, A\&A, 307, 791

Hammer, R. 1982, ApJ, 259, 767

Han, Z., Podsiadlowski, P., \& Eggleton, P. P. 1994, MNRAS, 270, 121

Hartigan, P., Edwards, S., \& Ghandour, L. 1995, ApJ, 452, 736

Hartigan, P., Edwards, S., \& Pierson, R. 2004, ApJ, 609, 261

Hartmann, L. 2000, Accretion Processes in Star Formation (Cambridge: Cambridge Univ. Press) 
Hartmann, L., Calvet, N., Gullbring, E., \& D’Alessio, P. 1998, ApJ, 495, 385

Hartmann, L., Cassen, P., \& Kenyon, S. J. 1997, ApJ, 475, 770

Hartmann, L., Hewett, R., \& Calvet, N. 1994, ApJ, 426, 669

Hartmann, L., \& MacGregor, K. B. 1980, ApJ, 242, 260

Heinemann, M., \& Olbert, S. 1980, J. Geophys. Res., 85, 1311

Höfner, S. 2005, in 13th Cambridge Workshop on Cool Stars, Stellar Systems, and the Sun, ed. F. Favata, G. Hussain, \& B. Battrick (Noordwijk, The Netherlands: ESA), ESA SP-560, 335

Hollweg, J. V. 1974, J. Geophys. Res., 79, 3845

Hollweg, J. V. 1976, J. Geophys. Res., 81, 1649

Hollweg, J. V. 1986, J. Geophys. Res., 91, 4111

Holzer, T. E., \& Axford, W. I. 1970, ARA\&A, 8, 31

Holzer, T. E., Flå, T., \& Leer, E. 1983, ApJ, 275, 808

Holzwarth, V. 2007, Mem. Soc. Astron. Italiana, 78, 271

Holzwarth, V., \& Jardine, M. 2007, A\&A, 463, 11

Hossain, M., Gray, P. C., Pontius, D. H., Jr., Matthaeus, W. H., \& Oughton, S. 1995, Phys. Fluids, 7, 2886

Illarionov, A. F., \& Sunyaev, R. A. 1975, A\&A, 39, 185

Jacques, S. A. 1977, ApJ, 215, 942

Jardine, M. M., Gregory, S. G., \& Donati, J.-F. 2008, MNRAS, 386, 688

Jeffries, R. D., Evans, P. A., Pye, J. P., \& Briggs, K. R. 2006, MNRAS, 367, 781

Johns, C. M., \& Basri, G. 1995, ApJ, 449, 341

Judge, P. G., Solomon, S. C., \& Ayres, T. R. 2003, ApJ, 593, 534

Käpylä, P. J., Korpi, M. J., Stix, M., \& Tuominen, I. 2007, in IAU Symp. 239, Convection in Astrophysics, ed. F. Kupka, I. Roxburgh \& K. Chan (Cambridge: Cambridge Univ. Press), 437

Killie, M. A. 2002, M.S. Thesis, University of Oslo, Norway

Klimchuk, J. A. 2006, Sol. Phys., 234, 41

Kohl, J. L., Noci, G., Cranmer, S. R., \& Raymond, J. C. 2006, A\&A Rev., 13, 31

Königl, A. 1991, ApJ, 370, L39

Königl, A., \& Pudritz, R. E. 2000, in Protostars and Planets IV, ed. V. Mannings, A. Boss, S. Russell (Arizona: Univ. Arizona Press), 759

Kulkarni, A. K., \& Romanova, M. M. 2008, MNRAS, 386, 673

Kuperus, M., Ionson, J. A., \& Spicer, D. S. 1981, ARA\&A, 19, 7

Kurucz, R. L. 1992, in IAU Symp. 149, The Stellar Populations of Galaxies, ed. B. Barbuy \& A. Renzini (Dordrecht: Kluwer), 225

Lada, C. J. 1985, ARA\&A, 23, 267

Lamzin, S. A. 1999, Astron. Lett., 25, 430

Lee, T., Shu, F. H., Shang, H., Glassgold, A. E., \& Rehm, K. E. 1998, ApJ, 506, 898

Leer, E., Hansteen, V. H., \& Holzer, T. E. 1998, in Cyclical Variability in Stellar Winds, ed. L. Kaper \& A. W. Fullerton (Berlin: Springer-Verlag), 263

Long, M., Romanova, M. M., \& Lovelace, R. V. E. 2007, MNRAS, 374, 436

Lucy, L. B. 1971, ApJ, 163, 95

Lucy, L. B. 1976, ApJ, 205, 482

Lynden-Bell, D., \& Pringle, J. E. 1974, MNRAS, 168, 603

Matt, S., \& Pudritz, R. E. 2005, ApJ, 632, L135

Matt, S., \& Pudritz, R. E. 2007, in IAU Symp. 243, Star-Disk Interactions in Young Stars, ed. J. Bouvier, I. Appenzeller (Cambridge: Cambridge U. Press), 299

Matt, S., \& Pudritz, R. E. 2008, ApJ, 681, 391

Matthaeus, W. H., Zank, G. P., Oughton, S., Mullan, D. J., \& Dmitruk, P. 1999, ApJ, 523, L93

Mazzotta, P., Mazzitelli, G., Colafrancesco, S., \& Vittorio, N. 1998, A\&AS, 133,403

McKee, C. F., \& Ostriker, E. C. 2007, ARA\&A, 45, 565

Mignone, A. 2005, ApJ, 626, 373

Mihalas, D. 1978, Stellar Atmospheres, 2nd ed. (San Francisco: W. H. Freeman)

Montalbán, J., D’Antona, F., Kupka, F., \& Heiter, U. 2004, A\&A, 416, 1081

Moreton, G. E., \& Ramsey, H. E. 1960, PASP, 72, 357

Mullan, D. J., \& Cheng, Q. Q. 1993, ApJ, 412, 312

Musielak, Z. E., \& Ulmschneider, P. 2002, A\&A, 386, 606

Muzerolle, J., Calvet, N., Briceño, C., Hartmann, L., \& Hillenbrand, L. 2000, ApJ, 535, L47

Muzerolle, J., Calvet, N., \& Hartmann, L. 2001, ApJ, 550, 944

Narain, U., \& Ulmschneider, P. 1990, Space Sci. Rev., 54, 377

Narain, U., \& Ulmschneider, P. 1996, Space Sci. Rev., 75, 453

Noyes, R. W., Hartmann, L. W., Baliunas, S. L., Duncan, D. K., \& Vaughan, A. H. 1984, ApJ, 279, 763

Oughton, S., Dmitruk, P., \& Matthaeus, W. H. 2006, Phys. Plasmas, 13, 042306
Owocki, S. P. 2004, in Evolution of Massive Stars, Mass Loss, and Winds, ed. M. Heydari-Malayeri, P. Stee, \& J.-P. Zahn, EAS Pub. Ser. 13, 163

Owocki, S. P., \& van Marle, A. J. 2008, in IAU Symp. 250, Massive Stars as Cosmic Engines, ed. F. Bresolin, P. Crowther, J. Puls (Cambridge: Cambridge U. Press), 71

Paatz, G., \& Camenzind, M. 1996, A\&A, 308, 77

Parker, E. N. 1953, ApJ, 117, 431

Parker, E. N. 1958, ApJ, 128, 664

Parker, E. N. 1963, Interplanetary Dynamical Processes (New York: Interscience)

Pols, O. R., Tout, C. A., Eggleton, P. P., \& Han, Z. 1995, MNRAS, 274, 964

Preibisch, T., \& Feigelson, E. D. 2005, ApJS, 160, 390

Raymond, J. C., Cox, D. P., \& Smith, B. W. 1976, ApJ, 204, 290

Reimers, D. 1975, Mem. Soc. R. Sci. Liège, 8, 369

Reimers, D. 1977, A\&A, 61, 217

Renzini, A., Cacciari, C., Ulmschneider, P., \& Schmitz, F. 1977, A\&A, 61, 39

Ribas, I., Guinan, E. F., Güdel, M., \& Audard, M. 2005, ApJ, 622, 680

Robinson, F. J., Demarque, P., Li, L. H., Sofia, S., Kim, Y.-C., Chan, K. L., \& Guenther, D. B. 2004, MNRAS, 347, 1208

Romaniello, M., Robberto, M., \& Panagia, N. 2004, ApJ, 608, 220

Romanova, M. M., Kulkami, A. K., \& Lovelace, R. V. E. 2008, ApJ, 673, L171

Rosner, R., An, C.-H., Musielak, Z. E., Moore, R. L., \& Suess, S. T. 1991, ApJ, 372, L91

Rosner, R., Musielak, Z. E., Cattaneo, F., Moore, R. L., \& Suess, S. T. 1995, ApJ, 442, L25

Rosner, R., Tucker, W. H., \& Vaiana, G. S. 1978, ApJ, 220, 643

Saar, S. H. 2001, in Cool Stars, Stellar Systems, and the Sun, 11th Cambridge Workshop, ed. R. Garcia Lopez, R. Rebolo, \& M. Zapaterio Osorio, ASP Conf. Ser. 223, 292

Safier, P. N., 1998, ApJ, 494, 336

Scheurwater, R., \& Kuijpers, J. 1988, A\&A, 190, 178

Schröder, K.-P., \& Cuntz, M. 2005, ApJ, 630, L73

Schwadron, N. A., McComas, D. J., \& DeForest, C. 2006, ApJ, 642, 1173

Shu, F. H., Galli, D., Lizano, S., \& Cai, M. J. 2007, in IAU Symp. 243, Star-Disk Interactions in Young Stars, ed. J. Bouvier, I. Appenzeller (Cambridge: Cambridge U. Press), 249

Skumanich, A. 1972, ApJ, 171, 565

Stempels, H. C. 2003, Ph.D. Dissertation, Uppsala Universitet, Sweden

Strom, K. M., Strom, S. E., Edwards, S., Cabrit, S., \& Skrutskie, M. F. 1989, AJ, 97, 1451

Struck, C., Smith, D. C., Willson, L. A., Turner, G., \& Bowen, G. H. 2004, MNRAS, 353, 559

Suzuki, T. K. 2006, ApJ, 640, L75

Suzuki, T. K. 2007, ApJ, 659, 1592

Telleschi, A., Güdel, M., Briggs, K. R., Audard, M., \& Palla, F. 2007, A\&A, 468,425

Thompson, B. J., Plunkett, S. P., Gurman, J. B., Newmark, J. S., St. Cyr, O. C., \& Michels, D. J. 1998, Geophys. Res. Lett., 25, 2465

Tu, C.-Y., \& Marsch, E. 1995, Space Sci. Rev., 73, 1

Uchida, Y. 1968, Sol. Phys., 4, 30

Uchida, Y., \& Shibata, K. 1984, PASJ, 36, 105

Ulmschneider, P., \& Muchmore, D. 1986, in Small Scale Magnetic Flux Concentrations in the Solar Photosphere, ed. W. Deinzer, M. Knölker, \& H. H. Voigt (Göttingen: Vandenhoeck \& Ruprecht), 191

Ustyugova, G. V., Koldoba, A. V., Romanova, M. M., \& Lovelace, R. V. E. 2006, ApJ, 646, 304

van Ballegooijen, A. A. 1994, Space Sci. Rev., 68, 299

Vasconcelos, M. J., Jatenco-Pereira, V., \& Opher, R. 2002, ApJ, 574, 847

Velli, M. 2001, Ap\&SS, 277, 157

Wang, Y.-M. 2000, ApJ, 543, L89

Wang, Y.-M., \& Sheeley, N. R., Jr. 1991, ApJ, 372, L45

Whang, Y. C. 1997, ApJ, 485, 389

Wills-Davey, M. J., DeForest, C. E., \& Stenflo, J. O. 2007, ApJ, 664, 556

Willson, L. A. 2000, ARA\&A, 38, 573

Withbroe, G. L. 1988, ApJ, 325, 442

Wood, B. E., Müller, H.-R., Zank, G. P., \& Linsky, J. L. 2002, ApJ, 574, 412

Wood, B. E., Müller, H.-R., Zank, G. P., Linsky, J. L., \& Redfield, S. 2005, ApJ, 628, L143

Zhou, Y., \& Matthaeus, W. H. 1990, J. Geophys. Res., 95, 10291 